\title{
Double Wells: Perturbation Series Summable to the Eigenvalues and Directly Computable Approximations
}

\author{
Emanuela Caliceti, Vincenzo Grecchi and Marco Maioli` \\ Dipartimento di Matematica, Università di Modena, I-41100 Modena, Italy
}

\begin{abstract}
We give a rigorous proof of the analyticity of the eigenvalues of the double-well Schrödinger operators and of the associated resonances. We specialize the Rayleigh-Schrödinger perturbation theory to such problems, obtaining an expression for the complex perturbation series uniquely related to the eigenvalues through a summation method. By an approximation we obtain new series expansions directly computable, still summable, which, in the case of the Herbst-Simon model, can be given in an explicit form.
\end{abstract}

\section{Introduction}

In many problems of physical interest the Rayleigh-Schrödinger perturbation theory provides divergent power series expansions for the eigenvalues. In several cases not only are such expansions asymptotic to the perturbed eigenvalues, but also the correspondence is one-to-one through summation methods such as the Borel one $([14,9,2])$. In other circumstances, like the Lo Surdo-Stark effect $([14,6])$, it has been proved that the Borel sum of the series in complex directions of the parameter defines the resonances of the problem.

As for the double-well Schrödinger operators, the situation is not completely satisfactory, since the perturbation series is summable in complex directions of the parameter to the "resonances" $([7,8])$ and thus the question is how to obtain the eigenvalues. This problem of perturbation theory could have connections in other areas, such as constructive field theory.

We believe we provide here the conclusive answer to this kind of problem with this type of techniques. In particular we define complex series expansions which are summable in complex directions of the parameter; the sums can be analytically continued to the real axis and are related to the eigenvalues in a simple way. Such series expansions are directly computable by means of approximations as we shall suggest. Better approximations can be obtained in the framework of this theory also by means of semiclassical methods.

In order to examine in more detail these questions, we restrict ourselves to

\footnotetext{
* Partially supported by Ministero della Pubblica Istruzione
} 
discussing two crucial examples, which will be the object of this work: (I) the first eigenvalue of the operator $H_{1}(g)=p^{2}+x^{2}(1-g x)^{2}+2 g x-1$ discussed by Herbst-Simon [10]; (II) any eigenvalue of the operator $H_{2}(g)=p^{2}+x^{2}(1-g x)^{2}$.

In case (I) we have stability, but all the perturbative coefficients are equal to zero so that the only regular sum of the power series is zero itself, while the eigenvalue is positive for $g>0$.

In case (II) the eigenvalue is unstable and splits into two eigenvalues for positive parameter. Nevertheless we overcome this problem by considering in the usual expression which defines the eigenvalue a $g$-dependent test vector with a definite parity with respect to $1 / 2 g$.

Before discussing the results we should say that usually Borel summability is first proved for the numerator and the denominator separately and afterwards extended to the eigenvalue by the algebraic properties of nonzero Borel sums ([11]). In this case, expecting a different kind of sum, we try to prove summability of a power series expansion of numerator and denominator separately. Of course such series depend on the test function that we use.

As a general result for the two problems, we have $E(g)=N(g) / D(g)$, where $N(g)=\operatorname{Re} F_{1}(g, g), D(g)=\operatorname{Re} F_{0}(g, g)$, and $F_{j}(g, \gamma), j=0,1$, is the Borel sum of the series $\Sigma_{n} C_{j, n}(g) \gamma^{n}$ for $g$, $|\gamma|$, arg $\gamma$ small positive, and is uniquely continuable in $\gamma$ to $\gamma=g$.

The coefficients $C_{j, n}(g)$ are complex: $C_{j, n}(g)=a_{j, n}(g)+i b_{j, n}(g)$, where $a_{j, n}(g)$ are the usual perturbation coefficients for a $g$-dependent test vector, and $b_{j, n}(g)$ are directly computable in the same way as one computes $a_{j, n}(g)$ only in the limit as $g \rightarrow 0^{+}$.

If we consider separetely the two series $\Sigma_{n} a_{j, n}(g) \gamma^{n}, \Sigma_{n} b_{j, n}(g) \gamma^{n}$ and call $\Phi_{j}^{R}(g, \gamma)$, $\Phi_{j}^{I}(g, \gamma)$ their Borel sums for $\operatorname{Im} \gamma>0$, we have that $\overline{\Phi_{j}^{R}(g, \bar{\gamma})}$ and $\overline{\Phi_{j}^{I}(g, \bar{\gamma})}$ are their sums for $\operatorname{Im} \gamma<0$ and $\operatorname{Re} F_{j}(g, g)=(1 / 2)\left(\Phi_{j}^{R}(g, \gamma)+\overline{\Phi_{j}^{R}(g, \bar{\gamma})}\right)+(i / 2)\left(\Phi_{j}^{I}(g, \gamma)-\right.$ $\left.\overline{\Phi_{j}^{I}(g, \bar{\gamma})}\right)\left.\right|_{\gamma=g>0}$, where the first term is the arithmetic mean of the two limiting values on the cut of a Borel sum, so that it can be a distributional Borel sum (DBS, see $[3,4]$ ) as we actually prove for model $(\mathrm{I})$, while the other one is the discontinuity of a Borel sum.

More explicitly in case (I) we have:

$$
E(g)=C_{1}(g) \operatorname{Im} \phi\left(g^{2}\right) /\left(\operatorname{Re} \phi\left(g^{2}\right)+C_{2}(g) \operatorname{Im} \phi\left(g^{2}\right)\right), \quad g>0,
$$

where $C_{1}(g), C_{2}(g)$ are analytic for $g>0$ and $C_{1}(g) \underset{g \rightarrow 0^{+}}{\rightarrow} 2 / \pi, C_{2}(g) \underset{g \rightarrow 0^{+}}{\rightarrow} 0$ and $\phi\left(g^{2}\right) \equiv \mathrm{e}^{\mathrm{i} \pi / 6} g^{5 / 3} \int_{-\infty}^{\infty} \exp \left(\left(2 i x^{3} / 3\right)-(i / g)^{2 / 3} x^{2}\right) d x \underset{g \rightarrow 0^{+}}{\sim} \sum_{n} a_{n} g^{2 n}$ is the Borel sum of $\sum_{n} a_{n} g^{2 n}$ for $\arg (g)$ small positive. In this case we can prove that $\operatorname{Re} \phi\left(g^{2}\right)$ is the DBS of $\sum_{n} a_{n} g^{2 n}$ on the positive real axis and $2 i \operatorname{Im} \phi\left(g^{2}\right)$ the associated "discontinuity" ([3,4]). Note that $\operatorname{Im} \phi\left(g^{2}\right) / \operatorname{Re} \phi\left(g^{2}\right)=A i\left((2 g)^{-4 / 3}\right) / B i\left((2 g)^{-4 / 3}\right)$.

We thus obtain the following explicit approximation coinciding with the asymptotic behavior

$$
\tilde{E}(g) \equiv(2 / \pi) \operatorname{Im} \phi\left(g^{2}\right) / \operatorname{Re} \phi\left(g^{2}\right) \sim \pi^{-1} \exp \left(-1 / 3 g^{2}\right) \sim E(g) \quad \text { as } \quad g \rightarrow 0 .
$$


We anticipate here that this method is based on a linear relationship between the matrix elements of the resolvant on dilation analytic vectors and the matrix elements of the resolvents of the "resonance" operators on the same vectors, suitably dilated (see (4.4)). As for the techniques, we specialize for double-well problems the stability arguments by Hunziker-Vock [15]. This way we rigorously prove the analyticity of the double-well eigenvalues (Sect. 2) as well as various stability properties of non-modal operators (Sect. 3) confirming, among other things, the results of [7]. We cannot, however, prove by these techniques the analyticity on the whole disc $\operatorname{Re} g^{-2}>c>0$, although it looks reasonable in case (II) (see [5]) and it is proved by direct inspection in case (I). In Sect. 4 we discuss the method and we give the results of the applications to these two problems.

\section{Double-Well Schrödinger Operators}

Let $g \in \mathbb{C} \backslash\{0\} ; H(g)$ will denote the operator in $L^{2}(\mathbb{R})$ defined by $D(H(g))=$ $D\left(p^{2}\right) \cap D\left(x^{4}\right)$,

$$
H(g) u \equiv H_{2}(g) u=\left(p^{2}+x^{2}(1-g x)^{2}\right) u \equiv\left(p^{2}+V(g)\right) u, \quad \forall u \in D(H(g)) .
$$

It is well-known (see [11]) that, for $g \in \mathbb{R}, H(g)$ is a self-adjoint operator with compact resolvent and thus with discrete spectrum. Moreover the eigenvalues $\left\{E_{n}\right\}_{n=1}^{\infty}$ of the harmonic oscillator $H(0)=p^{2}+x^{2}, D(H(0))=D\left(p^{2}\right) \cap D\left(x^{2}\right)$ are not stable with respect to the family $\{H(g)\}_{g>0}$. In fact $\forall n \in \mathbb{N}$ there exist two eigenvalues $E_{n}^{+}(g)$ and $E_{n}^{-}(g)$ of $H(g)$ which converge to $E_{n}$ as $g \rightarrow 0^{+}$. As for the case of complex $g$ one can prove with standard techniques based on quadratic estimates as in [13] that $H(g)$ is an analytic family of type $A$ in the open sector $|\arg (g)|<\pi / 4, g \neq 0$. As a consequence $H(g)$ has compact resolvent and discrete spectrum in the whole analyticity region. Similarly one can prove that for fixed $g, g \neq 0,|\arg (g)|<\pi / 4$, the operator

$$
H(\mu, g) \equiv \mu^{-2} p^{2}+\mu^{2} x^{2}(1-\mu g x)^{2}
$$

(which corresponds to the scaling $x \rightarrow \mu x$ ) when defined on $D\left(p^{2}\right) \cap D\left(x^{4}\right)$ represents an analytic family of type $A$ in $L^{2}(\mathbb{R})$ in $\mu$, for $|\arg (\mu)|<\pi / 4, \mu g>0$. In particular for such values of $\mu, H(\mu, g)$ has compact resolvent, discrete spectrum and its eigenvalues are independent of $\mu$, thus coinciding with those of $H(g)$.

Now let $g=\rho e^{i \theta}$, with $\rho=|g|>0$ and fixed $\theta:|\theta|<\pi / 4$. By choosing $\mu=e^{-i \theta}$ we pass from the operator $H(g)$ to the operator

$$
H(\mu, g)=e^{2 i \theta}\left(p^{2}+e^{-4 i \theta} V(\rho)\right) \equiv e^{2 i \theta} K(\rho),
$$

which, from the above remarks, has compact resolvent and discrete spectrum which coincides with $\sigma(H(g))$. We want to prove analogous properties to the self-adjoint case for the eigenvalues of $K(\rho), D(K(\rho))=D\left(p^{2}\right) \cap D\left(x^{4}\right)$, as $\rho \rightarrow 0^{+}$. To this end we will make large use of the symmetry of the potential $V(\rho)$ with respect to the barrier point $1 / 2 \rho$. More precisely let $P^{+}(\rho)\left(P^{-}(\rho)\right)$ be the operator on $L^{2}(\mathbb{R})$ which projects onto the even (odd) functions with respect to $1 / 2 \rho$, i.e.

$$
\left(P^{ \pm}(\rho) u\right)(x)=2^{-1}\left(u(x) \pm u\left(\rho^{-1}-x\right)\right), \quad \forall u \in L^{2}(\mathbb{R}) .
$$


$P^{ \pm}(\rho)$ are orthogonal projections, i.e. they are self-adjoint and idempotent, and the following properties are immediately verified:

(a) $P^{+}(\rho)+P^{-}(\rho)=I$ and thus $P^{+}(\rho) P^{-}(\rho)=P^{-}(\rho) P^{+}(\rho)=0$;

(b) $\left\|P^{ \pm}(\rho)\right\|=1$;

(c) $u \in \operatorname{Range}\left(P^{ \pm}(\rho)\right)$ if and only if $u(x)= \pm u\left(\rho^{-1}-x\right)$. In particular $V(\rho) \in$ Range $\left(P^{+}(\rho)\right)$;

(d) $P^{ \pm}(\rho) K(\rho) u=K(\rho) P^{ \pm}(\rho) u, \forall u \in D\left(p^{2}\right) \cap D\left(x^{4}\right)$.

Finally let us introduce the operators

$$
K^{ \pm}(\rho)=K(\rho) P^{ \pm}(\rho), \quad D\left(K^{ \pm}(\rho)\right)=D(K(\rho)) .
$$

It is easy to show that $\sigma(K(\rho))=\sigma\left(K^{+}(\rho)\right) \cup \sigma\left(K^{-}(\rho)\right) \backslash\{0\}$ and that $\sigma\left(K^{ \pm}(\rho)\right)$ consists of discrete eigenvalues and of the point $\lambda=0$ which belongs to the essential spectrum, since it is an eigenvalue of infinite multiplicity. Let now $K(0)$ denote the dilated harmonic oscillator defined by $K(0) u=\left(p^{2}+e^{-4 i \theta} x^{2}\right) u, \forall u \in D(K(0)) \equiv$ $D\left(p^{2}\right) \cap D\left(x^{2}\right)$. We shall prove the stability of the eigenvalues of $K(0)$ with respect to both $\left\{K^{+}(\rho)\right\}_{\rho>0}$ and $\left\{K^{-}(\rho)\right\}_{\rho>0}$. To this end we shall first show that

(i) if $\lambda \notin \sigma(K(0)) \cup\{0\}$, then $\lambda \in \Delta^{+} \cap \Delta^{-}$, where $\Delta^{ \pm}=\left\{z \in \mathbb{C}: z \notin \sigma\left(K^{ \pm}(\rho)\right)\right.$ and $\left(K^{ \pm}(\rho)-z\right)^{-1}$ is uniformly bounded as $\left.\rho \rightarrow 0^{+}\right\}$;

(ii) if $\lambda \in \sigma(K(0))$, let $r>0$ be sufficiently small so that the only eigenvalue of $K(0)$ enclosed in $\Gamma_{r}=\{z \in \mathbb{C}:|z-\lambda|=r\}$ is $\lambda$. Then $\operatorname{dim} Q^{ \pm}(\rho) \leqq \operatorname{dim} Q(0)$ as $\rho \rightarrow 0^{+}$, where

$$
Q^{ \pm}(\rho)=(2 \pi i)^{-1} \oint_{\Gamma_{r}}\left(z-K^{ \pm}(\rho)\right)^{-1} d z
$$

is the spectral projection of $K^{ \pm}(\rho)$ corresponding to the part of the spectrum enclosed in $\Gamma_{r} \subset \mathbb{C} \backslash \sigma\left(K^{ \pm}(\rho)\right)$. Similarly for $Q(0)$.

The proof is based on the stability theory developed by Hunziker-Vock in [15], which we shall follow in the basic steps, pointing out the changes whenever necessary. In order to complete the stability result we need also the following inequality:

(iii) $\operatorname{dim} Q^{ \pm}(\rho) \geqq \operatorname{dim} Q(0)$, as $\rho \rightarrow 0^{+}$.

It usually follows in a simple way from the strong convergence in the generalized sense, which however does not hold in this case, neither for $K^{+}(\rho)$ nor for $K^{-}(\rho)$. Our proof of (iii) will make use, instead, of the following

\section{Lemma 2.1.}

(a) $K(\rho) u \rightarrow K(0) u$ as $\rho \rightarrow 0^{+}, \forall u \in C_{0}^{\infty}(\mathbb{R})$.

(b) $\left\langle\left(K^{+}(\rho)-K^{-}(\rho)\right) u, v\right\rangle \rightarrow 0$, as $\rho \rightarrow 0^{+}, \forall u \in D\left(p^{2}\right) \cap D\left(x^{4}\right), \forall v \in L^{2}(\mathbb{R})$.

The same properties hold for the adjoint operators.

(c) $K^{ \pm}(\rho) u=K(\rho) u, \forall u \in D(K(\rho))$ such that $u=P^{ \pm}(\rho) u$.

Proof. Part (a) immediately follows from the convergence of $V(\rho)$ to $x^{2}$ as $\rho \rightarrow 0^{+}$, uniform on the compact subsets of $\mathbb{R}$. As for (b), it easily follows from (a) once we have proved that $\left(P^{+}(\rho)-P^{-}(\rho)\right) \stackrel{w}{\longrightarrow} 0$, as $\rho \rightarrow 0^{+}$. This is trivial, since for $u \in L^{2}(\mathbb{R})$ 
and $v \in C_{0}^{\infty}(\mathbb{R})$,

$$
\left\langle\left(P^{+}(\rho)-P^{-}(\rho)\right) u, v\right\rangle=\int_{\operatorname{supp}(v)} u\left(\rho^{-1}-x\right) \overline{v(x)} d x \rightarrow 0 \quad \text { as } \quad \rho \rightarrow 0^{+} .
$$

Part (c) is due to the fact that $P^{ \pm}(\rho)$ is idempotent.

We now start to examine (i) and (ii). To fix the ideas we shall work with the family $\left\{K^{+}(\rho)\right\}_{\rho>0}$, the case $\left\{K^{-}(\rho)\right\}_{\rho>0}$ being completely analogous. The following result refers to Example 5 of [15].

Lemma 2.2. There exist $\xi>0$ and $\gamma$, dependent only on $\theta$ such that

$$
\begin{gathered}
\left\langle u, p^{2} P^{+}(\rho) u\right\rangle \leqq \xi\left\{(\cos \gamma) \operatorname{Re}\left\langle u, K^{+}(\rho) u\right\rangle+(\sin \gamma) \operatorname{Im}\left\langle u, K^{+}(\rho) u\right\rangle\right\} \\
\forall u \in \mathbb{C}_{0}^{\infty}(\mathbb{R}), \quad \forall \rho>0 .
\end{gathered}
$$

Proof. Choosing $\gamma=-2 \theta$ we have

$$
\begin{aligned}
& \xi\left\{(\cos \gamma) \operatorname{Re}\left(p^{2}+e^{-4 i \theta} V(\rho)\right)+(\sin \gamma) \operatorname{Im}\left(p^{2}+e^{-4 i \theta} V(\rho)\right)\right\} \\
& \quad=\xi\left\{(\cos 2 \theta) p^{2}+V(\rho)(\cos 2 \theta)\right\} \geqq p^{2}
\end{aligned}
$$

if we take $\xi \geqq(\cos 2 \theta)^{-1}$, since $\cos 2 \theta>0, \forall \theta \in(-\pi / 4, \pi / 4)$. Thus, $\forall u \in C_{0}^{\infty}(\mathbb{R})$ $\xi\left\{(\cos \gamma) \operatorname{Re}\left\langle u,\left(p^{2}+e^{-4 i \theta} V(\rho)\right) u\right\rangle+(\sin \gamma) \operatorname{Im}\left\langle u,\left(p^{2}+e^{-4 i \theta} V(\rho)\right) u\right\rangle\right\} \geqq\left\langle u, p^{2} u\right\rangle$.

Now we need to insert the projection $P^{+}(\rho)$. We have

$$
\begin{aligned}
& \xi\left\{(\cos \gamma) \operatorname{Re}\left\langle u, K^{+}(\rho) u\right\rangle+(\sin \gamma) \operatorname{Im}\left\langle u, K^{+}(\rho) u\right\rangle\right\} \\
& \quad=\xi\left\{(\cos \gamma) \operatorname{Re}\left\langle P^{+}(\rho) u, K^{+}(\rho) P^{+}(\rho) u\right\rangle+(\sin \gamma) \operatorname{Im}\left\langle P^{+}(\rho) u, K^{+}(\rho) P^{+}(\rho) u\right\rangle\right\} \\
& \quad \geqq\left\langle P^{+}(\rho) u, p^{2} P^{+}(\rho) u\right\rangle=\left\langle u, p^{2} P^{+}(\rho) u\right\rangle,
\end{aligned}
$$

where we have used the fact that $P^{+}(\rho)$ is self-adjoint and idempotent, and it commutes with $p^{2}$ and $K(\rho)$. This concludes the proof of the lemma.

It follows from Lemma 2.2 that the numerical range of $K^{+}(\rho)$ is contained in a half-plane $Q$ independent of $\rho>0: Q=\{z \in \mathbb{C}:(\cos \gamma) \operatorname{Re}(z)+(\sin \gamma) \operatorname{Im}(z) \geqq 0\}$. Since the spectrum of $K^{+}(\rho)$ consists only of eigenvalues, $\sigma\left(K^{+}(\rho)\right) \subset Q, \forall \rho>0$. Then, $\forall z \notin Q$ we have $\left\|\left(z-K^{+}(\rho)\right)^{-1}\right\| \leqq\{\operatorname{dist}(z, Q)\}^{-1}$ and thus $z \in \Delta^{+}$.

As a consequence we have the following corollaries.

Corollary 2.3. $\Delta^{+} \neq \varnothing$.

Corollary 2.4. There exists $a>0$ independent of $\rho \rightarrow 0^{+}$such that

$$
\left\|\left(1+p^{2}\right)^{1 / 2} P^{+}(\rho) u\right\| \leqq a\left\{\left\|K^{+}(\rho) u\right\|+\left\|P^{+}(\rho) u\right\|\right\}, \quad \forall u \in C_{0}^{\infty}(\mathbb{R}) .
$$

In the next two lemmas we adapt Lemma 5.1 of [15] to the present situation.

Lemma 2.5. Let $\lambda \in \mathbb{C} \backslash\{0\}$. Suppose there exists a sequence $\left\{\rho_{n}, u_{n}\right\}$ such that $\rho_{n} \rightarrow 0^{+}$, $u_{n} \in D\left(K^{+}\left(\rho_{n}\right)\right),\left\|u_{n}\right\| \rightarrow 1$ and $\left\|\left(\lambda-K^{+}\left(\rho_{n}\right)\right) u_{n}\right\| \rightarrow 0$ as $n \rightarrow \infty$. Then there exists $a$ subsequence $\left\{\rho_{m(n)}, u_{m(n)}\right\}$ such that $\left\{\rho_{m(n)}, P^{+}\left(\rho_{m(n)}\right) u_{m(n)}\right\}$ enjoys the same properties, i.e. $\left\|P^{+}\left(\rho_{m(n)}\right) u_{m(n)}\right\| \rightarrow 1,\left\|\left(\lambda-K^{+}\left(\rho_{m(n)}\right)\right) P^{+}\left(\rho_{m(n)}\right) u_{m(n)}\right\| \rightarrow 0$ as $n \rightarrow \infty$.

Proof. Since $P^{+}(\rho)$ and $P^{-}(\rho)$ are orthogonal to each other and commute with 
$K^{+}(\rho)$ we have

$$
\begin{aligned}
0 & =\lim _{n \rightarrow \infty}\left\|\left(\lambda-K^{+}\left(\rho_{n}\right)\right) u_{n}\right\| \\
& =\lim _{n \rightarrow \infty}\left\{\left\|\left(\lambda-K^{+}\left(\rho_{n}\right)\right) P^{+}\left(\rho_{n}\right) u_{n}\right\|+\left\|\left(\lambda-K^{+}\left(\rho_{n}\right)\right) P^{-}\left(\rho_{n}\right) u_{n}\right\|\right\} \\
& \geqq \liminf _{n \rightarrow \infty}\left\|\left(\lambda-K^{+}\left(\rho_{n}\right)\right) P^{+}\left(\rho_{n}\right) u_{n}\right\|+\liminf _{n \rightarrow \infty}\left\|\lambda P^{-}\left(\rho_{n}\right) u_{n}\right\| .
\end{aligned}
$$

Thus, since $\lambda \neq 0$, there exists a subsequence $\left\{\rho_{m(n)}, u_{m(n)}\right\}$ such that $\left\|P^{-}\left(\rho_{m(n)}\right) u_{m(n)}\right\| \rightarrow 0$, whence $\left\|P^{+}\left(\rho_{m(n)}\right) u_{m(n)}\right\| \rightarrow 1$, as $n \rightarrow \infty$. Since $\left\|P^{+}\left(\rho_{m(n)}\right)\right\|=1$, we have $\left\|\left(\lambda-K^{+}\left(\rho_{m(n)}\right)\right) P^{+}\left(\rho_{m(n)}\right) u_{m(n)}\right\|=\left\|P^{+}\left(\rho_{m(n)}\right)\left(\lambda-K^{+}\left(\rho_{m(n)}\right)\right) u_{m(n)}\right\| \leqq$ $\left\|\left(\lambda-K^{+}\left(\rho_{m(n)}\right)\right) u_{m(n)}\right\|$. Since the third term tends to zero by hypothesis, so does the first one, and this completes the proof of the lemma.

Lemma 2.6. If $\lambda \notin \sigma(K(0)) \cup\{0\}$, then $\lambda \in \Delta^{+}$unless there exists a sequence $\left\{\rho_{n}, u_{n}\right\}$ such that

$$
\begin{aligned}
& \rho_{n} \rightarrow 0^{+}, \quad u_{n} \in D\left(K^{+}\left(\rho_{n}\right)\right), \quad u_{n}=P^{+}\left(\rho_{n}\right) u_{n^{\prime}}\left\|u_{n}\right\| \rightarrow 1, \quad u_{n} \stackrel{w}{\rightarrow} 0, \\
& \left\|\left(\lambda-K^{+}\left(\rho_{n}\right)\right) u_{n}\right\| \rightarrow 0, \quad \text { as } \quad n \rightarrow \infty .
\end{aligned}
$$

Proof. Suppose there exists $\varepsilon>0$ such that $\left\|\left(K^{+}(\rho)-\lambda\right) u\right\| \geqq \varepsilon\|u\|$ for all $\rho>0$ small enough and all $u \in D\left(K^{+}(\rho)\right)$. Since $\lambda \neq 0$ this implies $\lambda \notin \sigma\left(K^{+}(\rho)\right)$ and $\left\|\left(K^{+}(\rho)-\lambda\right)^{-1}\right\| \leqq \varepsilon^{-1}$, thus $\lambda \in \Delta^{+}$. Hence if $\lambda \notin \Delta^{+}$there exist two sequences $\rho_{n} \rightarrow 0^{+}$and $u_{n} \in D\left(K^{+}\left(\rho_{n}\right)\right),\left\|u_{n}\right\|=1$, such that $\left\|\left(\lambda-K^{+}\left(\rho_{n}\right)\right) u_{n}\right\| \rightarrow 0$. By Lemma 2.5 we may assume $u_{n}=P^{+}\left(\rho_{n}\right) u_{n}$, and by passing to a subsequence we may assume $u_{n} \stackrel{w}{\rightarrow} u$ as $n \rightarrow \infty$. Since $u_{n}=P^{+}\left(\rho_{n}\right) u_{n}$, it then follows from Lemma 2.1(a)-(c) that for all $v \in C_{0}^{\infty}(\mathbb{R})$,

$$
\begin{aligned}
0 & =\lim _{n \rightarrow \infty}\left\langle v,\left(\lambda-K^{+}\left(\rho_{n}\right)\right) u_{n}\right\rangle \\
& =\lim _{n \rightarrow \infty}\left\langle v,\left(\lambda-K\left(\rho_{n}\right)\right) u_{n}\right\rangle \\
& =\lim _{n \rightarrow \infty}\left\langle\left(\lambda-K\left(\rho_{n}\right)\right)^{*} v, u_{n}\right\rangle=\left\langle(\lambda-K(0))^{*} v, u\right\rangle .
\end{aligned}
$$

Since $C_{0}^{\infty}(\mathbb{R})$ is a core of $K(0)^{*}$, it follows that $u \in D(K(0))$ and $(\lambda-K(0)) u=0$. This implies $u=0$, because $\lambda \notin \sigma(K(0))$ by assumption.

Now we shall see how Weyl-type sequences can be obtained which are supported away from the two wells. More precisely we shall prove that Hypothesis 3 of [15] is satisfied.

Let $\chi \in C_{0}^{\infty}(\mathbb{R})$ with $\chi(x)=1$ for $|x| \leqq 1$ and $0 \leqq \chi(x) \leqq 1, \forall x \in \mathbb{R}$. For $n \in \mathbb{N}$ and $\rho>0$ let us define $\chi_{n}^{\rho}$ as follows. Let $\operatorname{supp}(\chi) \subset[-M, M]$ for some $M>0$, and let $\chi_{n}(x)=\chi(x / n)$. Then $\operatorname{supp}\left(\chi_{n}\right) \subset[-M n, M n]$.

(1) If $M n \leqq(2 \rho)^{-1}$, let

$$
\chi_{n}^{\rho}(x)= \begin{cases}\chi_{n}(x), & \text { if } x \leqq(2 \rho)^{-1} \\ \chi_{n}\left(\rho^{-1}-x\right), & \text { if } x \geqq(2 \rho)^{-1}\end{cases}
$$

(2) If $n \leqq(2 \rho)^{-1} \leqq M n$, let

$$
\chi_{n}^{\rho}(x)= \begin{cases}\chi_{n}(x), & \text { if } x \leqq n \\ 1, & \text { if } n \leqq x \leqq(2 \rho)^{-1} \\ \chi_{n}^{\rho}\left(\rho^{-1}-x\right), & \text { if } x \geqq(2 \rho)^{-1}\end{cases}
$$


(3) If $(2 \rho)^{-1} \leqq n$, again let

$$
\chi_{n}^{\rho}(x)= \begin{cases}\chi_{n}(x), & \text { if } x \leqq(2 \rho)^{-1} \\ \chi_{n}\left(\rho^{-1}-x\right), & \text { if } x \geqq(2 \rho)^{-1}\end{cases}
$$

By construction the function $\chi_{n}^{\rho}$ is in the range of $P^{+}(\rho)$, i.e. it is even with respect to $(2 \rho)^{-1}: P^{+}(\rho) \chi_{n}^{\rho}=\chi_{n}^{\rho}$. Now let $M_{n}(\rho)=1-\chi_{n}^{\rho}$. The function $M_{n}(\rho)$ is also in the range of $P^{+}(\rho)$ and supported away from the two wells, which are centered at $x=0$ and $x=\rho^{-1}$. In the next theorem we check that Hypothesis 3 of [15] is satisfied.

Theorem 2.7. For the function $M_{n}(\rho)$ defined above the following assertions hold:

(a) $M_{n}(\rho)$ is uniformly bounded in $n$ and $\rho$;

(b) if $\rho_{m} \rightarrow 0^{+}$and $u_{m} \in D\left(K^{+}\left(\rho_{m}\right)\right)$ with $\left\|u_{m}\right\| \rightarrow 1, u_{m}=P^{+}\left(\rho_{m}\right) u_{m}, u_{m} \stackrel{w}{\rightarrow} 0$ and $\left\|K^{+}\left(\rho_{m}\right) u_{m}\right\| \leqq$ (const) $\forall m$, then there exists $a>0$ such that

$$
\limsup _{m \rightarrow \infty}\left\|M_{n}\left(\rho_{m}\right) u_{m}\right\|>a>0 \quad \forall n
$$

(c) $M_{n}(\rho)$ maps $D\left(K^{+}(\rho)\right)$ into itself and $\lim _{n \rightarrow \infty}\left\|\left[M_{n}(\rho), K^{+}(\rho)\right]\left(z-K^{+}(\rho)\right)^{-1}\right\|=0$ uniformly in $\rho$, for some $z \in \Delta^{+}$.

Proof.

(a) It obviously follows from $\chi_{n}^{\rho}(x) \leqq 1, \forall x \in \mathbb{R}$.

(b) For any fixed $n$ we have

$$
\begin{aligned}
\left\|\chi_{n}^{\rho_{m}} u_{m}\right\|^{2} & =\lim _{m \rightarrow \infty} 2 \int_{-\infty}^{\left(2 \rho_{m}\right)-1}\left|\chi_{n}^{\rho_{m}}(x) u_{m}(x)\right|^{2} d x=2 \lim _{m \rightarrow \infty} \int_{-\infty}^{\left(2 \rho_{m}\right)-1}\left|\chi_{n}(x) u_{m}(x)\right|^{2} d x \\
& =2 \lim _{m \rightarrow \infty}\left\|\chi_{n} u_{m}\right\|^{2}=2 \lim _{m \rightarrow \infty}\left\|\chi_{n}\left(1+p^{2}\right)^{-1 / 2}\left(1+p^{2}\right)^{1 / 2} P^{+}\left(\rho_{m}\right) u_{m}\right\|^{2}=0 .
\end{aligned}
$$

The first equality follows from the fact that $\chi_{n}^{\rho_{m}} u_{m}$ is even with respect to $\left(2 \rho_{m}\right)^{-1}$; the second one follows from the definition of $\chi_{n}^{\rho_{m}}$ is the case $\left(2 \rho_{m}\right)^{-1} \geqq M n$. As for the third equality simply notice that $\chi_{n}(x)=0$ for $x \geqq M n$. Finally, to obtain that the limit is zero we have used the fact that the operator $\chi_{n}\left(1+p^{2}\right)^{-1 / 2}$ is compact and that $\left(1+p^{2}\right)^{1 / 2} P^{+}\left(\rho_{m}\right) u_{m} \stackrel{w}{\rightarrow} 0$. In fact, since $\left\{K^{+}\left(\rho_{m}\right) u_{m}\right\}$ is bounded by assumption it follows from Corollary 2.4 that $\left\{\left(1+p^{2}\right)^{1 / 2} P^{+}\left(\rho_{m}\right) u_{m}\right\}$ is bounded and thus weakly convergent to zero. Now (b) is proved since, in particular, limsup $\left\|M_{n}\left(\rho_{m}\right) u_{m}\right\|=1, \forall n \in \mathbb{N}$.

(c) Let us estimate the commutator $\left[M_{n}(\rho), K^{+}(\rho)\right]$. We have

$$
\left[M_{n}(\rho), p^{2}+e^{-4 i \theta} V(\rho)\right]=\left[\chi_{n}^{\rho}, p^{2}\right]=2 i n^{-1} \phi_{n}^{\rho} p-n^{-2} \psi_{n}^{\rho},
$$

where the functions $\phi_{n}^{\rho}$ and $\psi_{n}^{\rho}$, obtained by differentiating $\chi_{n}^{\rho}$ once and twice respectively, are uniformly bounded in $n$ and $\rho$. This is due to the fact that $\chi$ belongs to $C_{0}^{\infty}(\mathbb{R})$ and thus $d \chi / d x$ and $d^{2} \chi / d x^{2}$ are bounded on $\mathbb{R}$. Since from Corollary $2.4\left\|p P^{+}(\rho) u\right\| \leqq($ const $)\left(\left\|K^{+}(\rho) u\right\|+\|u\|\right)$, we have

$$
\left\|\left[M_{n}(\rho), K^{+}(\rho)\right] u\right\| \leqq c n^{-1}\left(\left\|K^{+}(\rho) u\right\|+\|u\|\right)
$$


$\forall u \in D\left(K^{+}(\rho)\right)$, for some constant $c>0$. Now (2.2) obviously implies (c) for all $z \in \Delta^{+}$

The following result corresponds to Lemma 5.3 of [15].

Lemma 2.8. If $\left\{\rho_{n}, u_{n}\right\}$ satisfies (2.1), then so does $\left\{\rho_{m}, v_{n}=M_{n}\left(\rho_{m}\right) u_{m}\right\}_{n \rightarrow \infty}$, provided that $m=m(n)$ is chosen sufficiently large for each $n$.

Theorem 2.9. For each $\lambda \in \mathbb{C}$ there exists $\delta>0$ such that

$d_{n}^{+}(\lambda, \rho) \equiv \inf \left\{\left\|\left(\lambda-K^{+}(\rho)\right) M_{n}(\rho) u\right\|: u \in D\left(K^{+}(\rho)\right),\left\|M_{n}(\rho) u\right\|=1, \quad P^{+}(\rho) u=u\right\} \geqq \delta$, for all $n>n_{0}$ and $\rho \rightarrow 0^{+}$.

Proof. First notice that $\left\|\left(\lambda-K^{+}(\rho)\right) M_{n}(\rho) u\right\| \geqq \operatorname{dist}\left(\lambda, E_{n}^{+}(\rho)\right)$, where $E_{n}^{+}(\rho)=$ $\left\{\left\langle M_{n}(\rho) u, K^{+}(\rho) M_{n}(\rho) u\right\rangle: u \in D\left(K^{+}(\rho)\right), P^{+}(\rho) u=u,\left\|M_{n}(\rho) u\right\|=1\right\}$. Since $E_{n}^{+}(\rho) \subset$ $\mathbb{R}^{+}+\left\{e^{-4 i \theta} V(\rho, x):|x|>n\right.$ and $\left.\left|x-\rho^{-1}\right|>n\right\}$, we only need to examine the numerical range of $K^{+}(\rho)$ at infinity and away from the two wells. Now the assertion follows from $\lim V(\rho, x)=+\infty$, which implies $\lim \operatorname{dist}\left(\lambda, E_{n}^{+}(\rho)\right)=+\infty$.

$$
\underset{\substack{|x| \rightarrow \infty \\ \rho \rightarrow 0^{+}}}{\substack{n \rightarrow \infty \\ \rho \rightarrow 0^{+}}}
$$

We are now ready to prove the above mentioned result (i).

Theorem 2.10. If $\lambda \notin \sigma(K(0)) \cup\{0\}$, then $\lambda \in \Delta^{+}$.

Proof. If $\lambda \notin \sigma(K(0))$ and $\lambda \neq 0$, by Lemma 2.6 there exists $\left\{\rho_{n}, u_{n}\right\}$ satisfying (2.1). From Lemma 2.8 there exists a subsequence $\left\{\rho_{m(n)}\right\}$ of $\left\{\rho_{n}\right\}$ such that $d_{n}^{+}\left(\lambda, \rho_{m(n)}\right) \rightarrow 0$ as $n \rightarrow \infty$, which contradicts Theorem 2.9.

In order to prove (ii) we need some further results.

Lemma 2.11. Let $\lambda \in \sigma(K(0))$ and $Q^{ \pm}(\rho)$ be defined as in (ii). Then Range $Q^{ \pm}(\rho) \subset$ Range $P^{ \pm}(\rho)$, for all $\rho \rightarrow 0^{+}$.

Proof. Let $\rho>0$ be fixed and sufficiently small, and $u=Q^{+}(\rho) u \in \operatorname{Range} Q^{+}(\rho)$. We want to show that $u=P^{+}(\rho) u$. We have

$$
P^{-}(\rho) u=(2 \pi i)^{-1} \oint_{\Gamma_{r}}\left(z-K^{+}(\rho)\right)^{-1} P^{-}(\rho) u d z
$$

and, setting $w(z)=\left(z-K^{+}(\rho)\right)^{-1} P^{-}(\rho) u \in D\left(K^{+}(\rho)\right)$, it follows that $P^{-}(\rho) u=$ $\left(z-K^{+}(\rho)\right) w(z)=z w(z)$, since $w(z)=P^{-}(\rho) w(z)$. Then from $(2.3)$ we have $P^{-}(\rho) u=$ $(2 \pi i)^{-1} \oint_{\Gamma_{r}} P^{-}(\rho) u z^{-1} d z=0$, since $\lambda \neq 0$, if $r$ is sufficiently small, and the claim is proved. The argument is completely analogous to show that Range $Q^{-}(\rho) \subset$ Range $P^{-}(\rho)$.

Lemma 2.12. Let $\lambda \in \sigma(K(0))$. If $\left\{\rho_{m}, u_{m}\right\}$ is a sequence such that $\rho_{m} \rightarrow 0^{+},\left\|u_{m}\right\|=1$, $Q^{+}\left(\rho_{m}\right) u_{m}=u_{m}$ and $u_{m} \stackrel{w}{\rightarrow} u$, then $u \neq 0$.

Proof. If we assume $u=0$ we obtain a contradiction proceeding exactly as in the proof of Theorem 5.4 of [15], thus we shall omit the details. Following the same argument we obtain

$$
\lim _{n \rightarrow \infty}\left(\limsup _{m \rightarrow \infty}\left\|M_{n}\left(\rho_{m}\right) u_{m}\right\|\right)=0 .
$$

On the other hand the sequence $K^{+}\left(\rho_{m}\right) u_{m}=K^{+}\left(\rho_{m}\right) Q^{+}\left(\rho_{m}\right) u_{m}$ is bounded because 
$K^{+}(\rho) Q^{+}(\rho)$ is uniformly bounded as $\rho \rightarrow 0^{+}$by Theorem 2.10. Then (2.4) contradicts Theorem 2.7(b), since $u_{m}=P^{+}\left(\rho_{m}\right) u_{m}$ by Lemma 2.11.

Finally we can prove the main result of this section.

Theorem 2.13. If $\lambda \in \sigma(K(0))$, then there exists $\rho_{0}>0$ such that $\operatorname{dim} Q^{+}(\rho) \leqq \operatorname{dim} Q(\rho)$ for all $\rho: 0<\rho<\rho_{0}$.

Proof. Suppose there exist sequences $\rho_{m} \rightarrow 0^{+}$and $u_{m} \in L^{2}(\mathbb{R}),\left\|u_{m}\right\|=1$, such that $Q^{+}\left(\rho_{m}\right) u_{m}=u_{m}$ and $Q(0) u_{m}=0$. By passing to a subsequence we may assume $u_{m} \stackrel{w}{\rightarrow} u$. Now let $Q(\rho)$ and $\Delta$ be defined as $Q^{+}(\rho)$ and $\Delta^{+}$respectively, with $K^{+}(\rho)$ replaced by $K(0)$. Then it is easy to see that $\Delta \supset \Delta^{+} \cap \Delta^{-}=\mathbb{C} \backslash(\sigma(K(0)) \cup\{0\})$. Hence it follows from Lemma 2.1(a) and Theorem 2.10 that $Q(\rho)$ converges strongly to $Q(0)$ as $\rho \rightarrow 0^{+}$, and similarly for the adjoint operators. Moreover, from Lemmas 2.11 and 2.1(c) we have $Q^{+}\left(\rho_{m}\right) u_{m}=Q\left(\rho_{m}\right) u_{m}=u_{m}$. Thus, $\forall v \in C_{0}^{\infty}(\mathbb{R})\left\langle u_{m}, v\right\rangle=$ $\left\langle Q\left(\rho_{m}\right) u_{m}, v\right\rangle=\left\langle u_{m}, Q\left(\rho_{m}\right)^{*} v\right\rangle \rightarrow\left\langle u, Q(0)^{*} v\right\rangle=\langle Q(0) u, v\rangle$, which implies $Q(0) u=u$. On the other hand one can easily see that $Q(0) u_{m} \stackrel{w}{\rightarrow} Q(0) u$, and thus $0=Q(0) u=u$. Now the contradiction immediately follows from Lemma 2.12 .

Before proving (iii) we make some remarks, in order to summarize the results obtained so far. First of all notice that $Q(\rho)=Q^{+}(\rho)+Q^{-}(\rho)$ easily follows from Lemmas 2.1(c) and 2.11. Next recall, as mentioned at the beginning of the section, that all the properties obtained so far, although stated and proved in many cases only for $K^{+}(\rho)$, hold also for $K^{-}(\rho)$. Since Range $Q^{+}(\rho)$ is orthogonal to Range $Q^{-}(\rho)$ we have

$$
\operatorname{dim} Q(\rho)=\operatorname{dim} Q^{+}(\rho)+\operatorname{dim} Q^{-}(\rho) .
$$

Moreover, since $Q(\rho) \stackrel{s}{\rightarrow} Q(0)$ as $\rho \rightarrow 0^{+}$, we have $\operatorname{dim} Q(\rho) \geqq \operatorname{dim} Q(0)$. Finally recall that $\sigma(K(0))$ consists of the sequence of simple eigenvalues $\left\{(2 n+1) e^{-2 i \theta}: n \in \mathbb{N}\right\}$, thus it follows from (2.5) and Theorem 2.13 that

$$
1 \leqq \operatorname{dim} Q^{+}(\rho)+\operatorname{dim} Q^{-}(\rho) \leqq 2, \quad \text { as } \quad \rho \rightarrow 0^{+} .
$$

Thus we cannot have both $\operatorname{dim} Q^{+}(\rho)=0$ and $\operatorname{dim} Q^{-}(\rho)=0$. In the next theorem we shall prove that $\operatorname{dim} Q^{+}(\rho)=\operatorname{dim} Q^{-}(\rho)$ as $\rho \rightarrow 0^{+}$, which, together with (2.5) and (2.6), implies that

$$
\left.\begin{array}{rl}
\operatorname{dim} Q^{+}(\rho)= & \operatorname{dim} Q^{-}(\rho)=\operatorname{dim} Q(0)=1 \\
& \operatorname{dim} Q(\rho)=2
\end{array}\right\} \text { as } \quad \rho \rightarrow 0^{+} .
$$

In other words, in any neighbourhood of a given eigenvalue $\lambda$ of $K(0)$ there are exactly two distinct eigenvalues $\lambda^{+}(\rho)$ and $\lambda^{-}(\rho)$ of $K(\rho)$ (the former of $K^{+}(\rho)$ and the latter of $K^{-}(\rho)$ ), both converging to $\lambda$ as $\rho \rightarrow 0^{+}$. In order to complete our stability result we need the following

Lemma 2.14. For each $z \in \Delta^{+}=\Delta^{-}, \quad\left\{\left(K^{+}(\rho)-z\right)^{-1}-\left(K^{-}(\rho)-z\right)^{-1}\right\}^{W} \rightarrow 0$, as $\rho \rightarrow 0^{+}$, and similarly for the adjoint operators.

Proof. Let $u, v \in C_{0}^{\infty}(\mathbb{R})$. Then, using the second resolvent formula, the fact that $K^{+}(\rho)$ and $K^{-}(\rho)$ commute and the identity $K(\rho)=K^{+}(\rho)+K^{-}(\rho)$, we obtain $\left\langle\left\{\left(K^{+}(\rho)-z\right)^{-1}-\left(K^{-}(\rho)-z\right)^{-1}\right\} u, v\right\rangle=\left\langle z^{-1}(z-K(\rho))^{-1}\left(K^{-}(\rho)-K^{+}(\rho)\right) u, v\right\rangle$. Now it follows from Lemma 2.1(a) that $\left\{(z-K(\rho))^{-1}\right\}^{*}$ converges strongly to 
$\left\{(z-K(0))^{-1}\right\}^{*}$ as $\rho \rightarrow 0^{+}$. Since $C_{0}^{\infty}(\mathbb{R})$ is dense in $L^{2}(\mathbb{R})$, the assertion is a consequence of Lemma 2.1(b).

Theorem 2.15. Let $\lambda \in \sigma(K(0))$. Then there exists $\rho_{0}>0$ such that $\operatorname{dim} Q^{+}(\rho)=$ $\operatorname{dim} Q^{-}(\rho)$ for all $\rho \in\left(0, \rho_{0}\right)$.

Proof. Suppose there exists a sequence $\rho_{n} \rightarrow 0^{+}$such that $\operatorname{dim} Q^{+}\left(\rho_{n}\right)=1>0=$ $\operatorname{dim} Q^{-}\left(\rho_{n}\right)$. Then there exists $\left\{u_{n}\right\} \subset L^{2}(\mathbb{R})$ with $\left\|u_{n}\right\|=1$ and $u_{n}=Q^{+}\left(\rho_{n}\right) u_{n}$. By passing to a subsequence, if necessary, we may assume $u_{n} \stackrel{w}{\rightarrow} u$ and, by Theorem 2.12, $u \neq 0$. Since $u_{n}=P^{+}\left(\rho_{n}\right) u_{n}$, by Lemma $2.1(\mathrm{c}) u_{n}=Q\left(\rho_{n}\right) u_{n}$. Since $Q\left(\rho_{n}\right)^{*} \stackrel{s}{\rightarrow} Q(0)^{*}$, we have $u_{n} \stackrel{w}{\rightarrow} Q(0) u$, and thus $u=Q(0) u$. Moreover $Q\left(\rho_{n}\right)=Q^{+}\left(\rho_{n}\right)+Q^{-}\left(\rho_{n}\right)=$ $Q^{+}\left(\rho_{n}\right)$, because $Q^{-}\left(\rho_{n}\right) \equiv 0$, for each $n$. Now we obtain a contradiction. In fact, on one hand $\left\langle\left(Q^{+}\left(\rho_{n}\right)-Q^{-}\left(\rho_{n}\right)\right) u, u\right\rangle \rightarrow 0$ as $n \rightarrow \infty$ by Lemma 2.14. On the other hand $\left\langle\left(Q^{+}\left(\rho_{n}\right)-Q^{-}\left(\rho_{n}\right)\right) u, u\right\rangle=\left\langle Q\left(\rho_{n}\right) u, u\right\rangle \rightarrow\langle Q(0) u, u\rangle=\langle u, u\rangle \neq 0$. Similarly one can show there cannot exist a sequence $\rho_{n} \rightarrow 0^{+}$such that $\operatorname{dim} Q^{-}\left(\rho_{n}\right)=1$ and $\operatorname{dim} Q^{+}\left(\rho_{n}\right)=0$. This result combined with (2.6) proves the assertion.

We conclude this section with the following

Remark 2.16. All the results obtained in this section, in particular the stability of the eigenvalues of $K(0)$ with respect to the family $\left\{K^{ \pm}(\rho)\right\}_{\rho>0}$, hold uniformly in $\theta$, in any compact interval $|\theta| \leqq \theta_{0}, \theta_{0} \in(0, \pi / 4)$.

\section{Associated Operators}

In the present section some non-modal operators are studied, which are associated with the Schrödinger equations considered. We want to obtain in a rigorous way the stability of "resonances" (Theorem 3.5) already introduced in [7], and we want to prove the convergence (as $g \rightarrow 0$ ) of some ratio of Wronskians (Theorems 3.6, 3.13). In particular, we start off with case (II).

In the following we denote by $\sigma_{p}(A)$ the set of isolated eigenvalues of finite multiplicity of the operator $A$, and by $\sigma_{\text {ess }}(A)$ the set $\sigma(A) \backslash \sigma_{p}(A)$.

Lemma 3.1. Let $\rho_{0}>0$ be fixed and $0 \leqq \rho<\rho_{0}$. Let $A(\rho)$ be operators in $L^{2}(\mathbb{R})$ obtained as the closure of the differential expressions $f(x)^{2} p^{2}-i f^{\prime}(x) f(x) p+V_{\rho}(x)$ on $C_{0}^{\infty}(\mathbb{R})$, with $V_{\rho} \in L_{\text {loc }}^{2}(\mathbb{R}), f \in L^{\infty}(\mathbb{R}) \cap H^{1}(\mathbb{R})$. Let $V_{\rho} \rightarrow V_{0}$ as $\rho \rightarrow 0$ in the $L_{\text {loc }}^{2}(\mathbb{R})$ topology. Suppose that, for some $c>0$ independent of $\rho$,

$$
\left\|\left(1+p^{2}\right)^{1 / 2} u\right\| \leqq c(\|u\|+\|A(\rho) u\|), \quad \forall u \in C_{0}^{\infty}(\mathbb{R}) .
$$

Suppose that $\bigcup_{\rho \geqq 0} \sigma_{\text {ess }}(A(\rho)) \neq \mathbb{C}$ and let $z \notin \bigcup_{\rho \geqq 0} \sigma_{\text {ess }}(A(\rho))$. Moreover, let there exist intervals $\left(a_{j}(n, \rho), b_{j}(n, \rho)\right)\left(j= \pm 1, \pm 2, \ldots, \pm r ; n \in \mathbb{N} ; 0 \leqq \rho \leqq \rho_{0}\right)$ of length greater than $2 n$ satisfying

$$
\begin{aligned}
n= & a_{1}(n, \rho)<b_{1}(n, \rho)=a_{2}(n, \rho)+n<b_{2}(n, \rho)=a_{3}(n, \rho)+n<\cdots<b_{r}=+\infty, \\
& -\infty=a_{-r}<\cdots<b_{-1}(n, \rho)=-n, \quad\{x:|x|>n\}=\bigcup_{j}\left(a_{j}(n, \rho), b_{j}(n, \rho)\right),
\end{aligned}
$$

and let $E_{j}(n, \rho)=\left\{\langle u, A(\rho) u\rangle: u \in C_{0}^{\infty}(\mathbb{R}),\|u\|=1\right.$, supp $\left.u \subset\left(a_{j}(n, \rho), b_{j}(n, \rho)\right)\right\}$ satisfy the following property: there is $\left\{\sigma_{n}\right\} \rightarrow 0$ such that, for any sequence $\sigma_{n}^{\prime}=O\left(\sigma_{n}\right)$ one 
can find $\delta>0$ so that

$$
\operatorname{dist}\left(z, E_{j}\left(n, \sigma_{n}^{\prime}\right)\right) \geqq \delta>0 \quad \text { for } n \text { large enough. }
$$

If $z \notin \sigma_{p}(A(0))$, then $z \in \Delta \equiv\left\{w \in \mathbb{C}: w \notin \sigma(A(\rho))\right.$ and $[A(\rho)-w]^{-1}$ is uniformly bounded for small $\rho \geqq 0\}$. In particular $\Delta \neq \varnothing$.

Proof. After fixing $\chi \in C_{0}^{\infty}(\mathbb{R}), 0 \leqq \chi \leqq 1, \chi(x)=1$ for $|x| \leqq 1$ and $\chi(x)=0$ for $|x| \geqq 2$, we set $M_{n}(x)=1-\chi_{n}(x)=1-\chi(x / n)$. We also define $M^{j}(n, \rho) \in C^{\infty}(\mathbb{R})$ (for all $j, n$ and $\rho$ ) so that

$$
\begin{array}{r}
\operatorname{supp} M^{j}(n, \rho) \subset\left(a_{j}(n, \rho), b_{j}(n, \rho)\right), M_{n}(x)=\sum_{j=-r, j \neq 0}^{r} M^{j}(n, \rho)(x), \\
\left|d^{k} / d x^{k}\left(M^{j}(n, \rho)(x)\right)\right| \leqq n^{-k} \sup _{x}\left|d^{k} \chi(x) / d x^{k}\right| .
\end{array}
$$

One way of defining $M^{j}(n, \rho)$ is the following: for $j=1,2, \ldots, r-1$ let

and

$$
M^{j}(n, \rho)(x)= \begin{cases}0, & x \notin\left(a_{j}(n, \rho), b_{j}(n, \rho)\right) \\ 1, & x \in\left(a_{j}(n, \rho)+n, b_{j}(n, \rho)-n\right) \\ \chi\left(n^{-1}\left[x-b_{j}(n, \rho)+2 n\right]\right), & b_{j}(n, \rho)-n \leqq x \leqq b_{j}(n, \rho) \\ 1-\chi\left(n^{-1}\left[x-a_{j}(n, \rho)+n\right]\right), & a_{j}(n, \rho) \leqq x \leqq a_{j}(n, \rho)+n\end{cases}
$$

$$
M^{r}(n, \rho)(x)= \begin{cases}0, & x<a_{r}(n, \rho) \\ 1-\chi\left(n^{-1}\left[x-a_{r}(n, \rho)+n\right]\right), & x \geqq a_{r}(n, \rho) .\end{cases}
$$

Similar definitions for $j<0$.

Taking into account these definitions the lemma is proved "ab absurdo." Since $z \notin \bigcup \sigma_{\text {ess }}(A(\rho))$, by Lemma 5.1 of [15] we have $z \in \Delta$ unless there are two sequences $\left\{\rho_{m}\right\} \subset 0 \mathbb{R}_{+},\left\{u_{m}\right\} \subset D\left(A\left(\rho_{m}\right)\right)$ such that

$$
\rho_{m} \rightarrow 0, \quad\left\|u_{m}\right\| \nrightarrow 0, \quad u_{m} \stackrel{w}{\longrightarrow} 0, \quad\left\|\left(z-A\left(\rho_{m}\right)\right) u_{m}\right\| \rightarrow 0 .
$$

Since $C_{0}^{\infty}(\mathbb{R})$ is a core of $A(\rho)$, we can choose $\left\{u_{m}\right\}$ in $C_{0}^{\infty}(\mathbb{R})$. Since, by $(3.1),\{(1+$ $\left.\left.p^{2}\right)^{1 / 2} u_{m}\right\}$ is bounded, it tends weakly to zero, whence $\| \chi_{n}\left(1+p^{2}\right)^{-1 / 2}$. $\left(1+p^{2}\right)^{1 / 2} u_{m} \| \rightarrow 0$ as $m \rightarrow \infty$. As a consequence, for each $n, \lim _{m}\left\|M_{n} u_{m}\right\|=1$. So

$$
\underset{m}{\limsup }\left\|M^{j}(n, \rho) u_{m}\right\| \geqq(2 r)^{-1}>0 \quad \text { at least for one } j
$$

for each $n \in \mathbb{N}$, uniformly in $\rho\left(j= \pm 1, \pm 2, \ldots, \pm r ; 0<\rho<\rho_{0}\right)$. Besides, by (3.1), (3.3) and by conditions on $f(x)$ we have:

$$
\left\|\left[M^{j}(n, \rho), A(\rho)\right] u\right\| \leqq c_{0} n^{-1}(\|A(\rho) u\|+\|u\|)
$$

with $c_{0}$ independent of $n$ and $\rho$.

Therefore, if $\left\{\rho_{m}\right\}$ satisfies (3.4) then by (3.5) and (3.6) there is $m=m(n)$ such that $\rho_{m(n)} \rightarrow 0,\left\|M^{j}\left(n, \rho_{m(n)}\right) u_{m(n)}\right\| \rightarrow 0$ for at least one $j$,

$$
\begin{aligned}
& M^{j}\left(n, \rho_{m(n)}\right) u_{m(n)} \stackrel{w}{\longrightarrow} 0 \text { and }\left\|\left\{z-A\left(\rho_{m(n)}\right)\right\} M^{j}\left(n, \rho_{m(n)}\right) u_{m(n)}\right\| \\
& \quad \leqq\left(\left\|\left\{z-A\left(\rho_{m(n)}\right)\right\} u_{m(n)}\right\|+\left\|\left[M^{j}\left(n, \rho_{m(n)}\right), A\left(\rho_{m(n)}\right)\right] u_{m(n)}\right\|\right) \rightarrow 0 \quad \text { as } n \rightarrow \infty .
\end{aligned}
$$


By passing to a subsequence we may assume that $\rho_{m(n)}=O\left(\sigma_{n}\right)$ so that by hypothesis (3.2) we have the contradiction

$$
\left\|\left\{z-A\left(\rho_{m(n)}\right)\right\} M^{j}\left(n, \rho_{m(n)}\right) u_{m(n)}\right\| \geqq \operatorname{dist}\left(z, E_{j}\left(n, \rho_{m(n)}\right)\right) \geqq \delta>0
$$

for $n$ large. This proves the lemma.

Theorem 3.2. Let the hypotheses of the preceding lemma be verified except for the following modifications:

(i) $\operatorname{dist}\left(z, \sigma_{\text {ess }}(A(\rho))\right) \geqq \delta^{\prime}$, for some $\delta^{\prime}>0,0 \leqq \rho \leqq \rho_{0}$,

(ii) $z \in \sigma_{p}(A(0))$ (instead of: $z \notin \sigma_{p}(A(0))$ ).

Then $z$ is a stable eigenvalue of $A(0)$ with respect to the family $A(\rho)$ as $\rho \rightarrow 0$.

Proof. By Lemma 3.1 any $\lambda \neq z$ of a convenient neighbourhood of $z$ belongs to $\Delta$, so that $\Delta \neq \varnothing$. From this fact and from the construction of $M^{j}(n, \rho)$ in the proof of Lemma 3.1 (in particular from (3.5) and (3.6)) it follows that the operator family $A(\rho)$ and the associated operators $M^{j}(n, \rho)$ satisfy Hypothesis 3 of [15]. Under these conditions, according to the stability criterion stated in [15], Theorem 5.8, the needed estimate would be

$$
\begin{aligned}
d_{n}^{(j)}(z, \rho) \equiv & \inf \left\{\left\|(z-A(\rho)) M^{j}(n, \rho) u\right\|: \quad M^{J}(n, \rho) u \in D(A(\rho)),\right. \\
& \left.\cdot\left\|M^{j}(n, \rho) u\right\|=1\right\} \geqq \delta>0
\end{aligned}
$$

uniformly in $n$ and $\rho$, for $j= \pm 1, \pm 2, \ldots, \pm r$.

Now, by (3.2) we have that $d_{n}^{(j)}\left(z, \rho_{n}^{\prime}\right) \geqq \delta \geqq 0(j= \pm 1, \ldots, \pm r)$ for any sequence $\left\{\rho_{n}^{\prime}\right\}$ tending to zero sufficiently fast. This is sufficient to ensure stability, as it turns out by the same arguments used in [15, Theorem 5.4]. This completes the proof.

Let us consider the "resonance" operators defined in [7] as the closed operators given by

$$
H^{( \pm)}(g)=e^{-\imath( \pm \pi-2 \theta) / 3}\left\{p^{2}+x^{2}\left(e^{i( \pm \pi-2 \theta) / 3}-|g| e^{ \pm i \pi / 2} x\right)\right\}
$$

and having $C^{2}(\mathbb{R}) \cap L^{2}(\mathbb{R})$ as a core, with $\theta=\arg (g),-\pi / 4<\theta<5 \pi / 4$ for $H^{(+)}(g)$ and $-5 \pi / 4<\theta<\pi / 4$ for $H^{(-)}(g)$. $H^{( \pm)}(g)$ is the realization in $L^{2}(\mathbb{R})$ through complex scaling of the eigenvalue problem $\left(p^{2}+x^{2}(1-g x)^{2}\right) \psi=z \psi$, when we look for eigenfunctions exponentially decaying along the line $\mathbb{R} e^{ \pm i \pi / 6}$ if $g \in \mathbb{R}$, as $|x| \rightarrow \infty$ (along the line $\mathbb{R} e^{l(-\theta \pm \pi / 2) / 3}$ if $\theta \neq 0$ ).

To fix ideas, let us consider $H^{(-)}(g)$ and set $\alpha=-(\theta+\pi / 2) / 3$. By the standard Liouville-Green approximation one checks that the solutions keep their $L^{2}$ properties if $x$ is replaced by $x e^{-i \bar{\eta}}$ for $x \gg 0$ and by $x e^{i \bar{\eta}}$ for $x \ll 0(0<\bar{\eta} \ll 1)$ : see (9) in [7] and [12, par.6, 21, 24]. Hence, choosing a non-increasing $\eta \in C^{\infty}(\mathbb{R})$ such that $\eta(x)=\bar{\eta}$ for $x \leqq-3$ and $\eta(x)=-\bar{\eta}$ for $x \geqq 3$, we have a deformation:

$$
\phi \rightarrow \tilde{\phi}, \quad \tilde{\phi}(x)=\phi(\xi(x))\left(\xi^{\prime}(x)\right)^{1 / 2}, \quad \xi(x)=x e^{i \eta(x)}
$$

which transforms $H^{(-)}(g)$ into a compact resolvent operator with the same eigenvalues.

Explicitly, factoring $e^{i(2 \theta+\pi) / 3}, H^{(-)}(g)$ is transformed into

$$
\tilde{H}^{(-)}(g)=f(x)^{2} p^{2}-i f^{\prime}(x) f(x) p-\xi(x)^{2}\left(e^{i(-2 \theta+\pi / 2) / 3}-\rho \xi(x)\right)^{2},
$$

where $g=\rho e^{i \theta}, \rho \geqq 0$, and 


$$
f(x)=\left(\xi^{\prime}(x)\right)^{-1}
$$

Now we prove that, for some dense set of dilation analytic functions (such as $\mathscr{C}=\left\{\psi: \psi(x)=e^{-x^{2} / 2} P(x), P(x)\right.$ is a polynomial $\left.\}\right)$,

$$
\left\langle\left[H^{(-)}(g)-z\right]^{-1} \phi_{-\alpha}, \psi_{\alpha}\right\rangle=\left\langle\left[e^{i(\pi+2 \theta) / 3} \tilde{H}^{(-)}(g)-z\right]^{-1} \tilde{\phi}_{-\alpha}, \tilde{\psi}_{\alpha}\right\rangle,
$$

where $\phi, \psi \in \mathscr{C}, \phi_{\alpha}(x)=e^{i \alpha / 2} \phi\left(e^{i \alpha} x\right), \tilde{\phi}_{\alpha}(x)=\phi_{\alpha}(\xi(x))\left(\xi^{\prime}(x)\right)^{1 / 2}$, with $|\alpha \pm \bar{\eta}|<\pi / 4$. Indeed this equality is obtained by a deformation of the real axis into the integration path defined by $\xi(x)$ and using Cauchy's theorem and the fast decay of the integrand in the whole sector between $\pi$ and $\pi+\bar{\eta}$ and in the sector between 0 and $-\bar{\eta}$. This proves (3.13), so we can show the spectral properties and the estimates relative to $H^{(-)}(g)$ by studying $\tilde{H}^{(-)}(g)$.

Notice that, for $g=0$, this construction leads to an operator $\tilde{H}^{(-)}(0)$ which has the same spectrum of the usual harmonic oscillator (up to the above phase factor). We want to prove that the eigenvalues of $\widetilde{H}^{(-)}(0)$ are stable with respect to the family $\tilde{H}^{(-)}(g)$ as $\rho \rightarrow 0$, for $-5 \pi / 4+\varepsilon \leqq \theta \leqq \pi / 4-\varepsilon$, for any fixed $\varepsilon>0$, choosing $0<\bar{\eta} \ll \varepsilon$. For sake of simplicity we prove the intermediate steps only for $-\pi / 2 \leqq \theta \leqq \pi / 4-\varepsilon$.

Proposition 3.3. For any fixed $\varepsilon>0, \theta \in[-\pi / 2, \pi / 4-\varepsilon]$ and $\bar{\eta}>0$ sufficiently small with respect to $\varepsilon$, the operator $\tilde{H}^{(-)}(\mathrm{g})$ satisfies assumption (3.1) of Lemma 1.

Proof. Setting $\beta=\pi / 4-\theta, V_{\rho}(x)=-\xi(x)^{2}\left(e^{2 i \beta / 3}-\rho \xi(x)\right)^{2}, \beta \in[\varepsilon, 3 \pi / 4], \quad$ it $\quad$ is enough to find a real-valued function $\gamma_{\rho}(x), x \in \mathbb{R}$, such that

$$
\operatorname{Re}\left\{\exp \left(-i \gamma_{\rho}(x)\right)\left(f(x)^{2} \mu^{2}-i f^{\prime}(x) f(x) \mu+V_{\rho}(x)\right)\right\}+k \geqq c \mu^{2},
$$

where $k, c>0$ are independent of $x, \mu$ and $\rho\left(\mu \in \mathbb{R}, 0 \leqq \rho<\rho_{0}\right)$. Since $\eta(x)= \pm \bar{\eta}$, $f(x)=e^{ \pm i \bar{\eta}}$ for $|x| \geqq 3$, choosing $\gamma_{\rho}(x)=(1 / 2)\left(\arg V_{\rho}(x)+2 \arg f(x)\right)$ one checks that

$$
\operatorname{Re}\left\{\exp \left(-i \gamma_{\rho}(x)\right) V_{\rho}(x)\right\}+k_{1} \geqq 0
$$

for some $k_{1}>0$ : indeed $V_{\rho}$ is bounded for $|x| \leqq 3$. Moreover there is $c_{0}>0$ such that

$$
\operatorname{Re}\left\{\exp \left(-i \gamma_{\rho}(x)\right) f(x)^{2} \mu^{2}\right\} \geqq c_{0} \mu^{2}, \quad \forall x \in \mathbb{R}
$$

Indeed one can take $c_{0}=\sin \bar{\eta}$ for $|x|>3$. For $|x| \leqq 3$ one can find $\rho_{0}>0$ so that, if $0<\rho<\rho_{0}, V_{\rho}(x)$ is close to $-e^{4 i \beta / 3} \xi(x)^{2}$, whose phase is $-\pi+4 \beta / 3+2 \eta(x)$, with $|\eta(x)| \leqq \bar{\eta}$ : this allows us to find $c_{0}>0$ satisfying (3.16). Now (3.14) is a consequence of (3.15) and (3.16): indeed the term - if $(x) f(x) \mu(\mu \in \mathbb{R})$ is easily checked not to modify the asymptotic behaviour of $f(x)^{2} \mu^{2}+V_{\rho}(x)$ as $|\mu| \rightarrow \infty$. This proves the assertion.

Proposition 3.4. If $z \in \mathbb{C} \backslash \sigma_{p}\left(\tilde{H}^{(-)}(0)\right)$, then the resolvents $\left(\tilde{H}^{(-)}(g)-z\right)^{-1}$ exist for $|g|$ small and are uniformly bounded as $|g| \rightarrow 0$. If $z \in \sigma_{p}\left(H^{(-)}(0)\right)$ then it is a stable eigenvalue with respect to the family $\tilde{H}^{(-)}(g)$.

Proof. The estimate (3.1) is satisfied by $\tilde{H}^{(-)}(\mathrm{g})$ by virtue of Proposition 3.3 while $\sigma_{\text {ess }}\left(\tilde{H}^{(-)}(g)\right)=\varnothing, \forall g$. Therefore it is enough to verify, $\forall z \in \mathbb{C}$, the second group of hypotheses of Lemma 3.1, in particular (3.2); then the first assertion will follow from Lemma 3.1 and the second assertion will be true by Theorem 3.2. 
Suppose $\beta=\pi / 4-\theta, \beta \in[\varepsilon, 3 \pi / 4]$ (analogous arguments hold for $\beta>3 \pi / 4$ ). Define, for each $n \in \mathbb{N}$ and $0<\rho<1 / 2 n: a_{1}(n, \rho)=n, b_{1}(n, \rho)=2 n+1 / \rho, a_{2}(n, \rho)=$ $n+1 / \rho, b_{2}(n, \rho)=+\infty$. Let $a_{-1}, b_{-1}, a_{-2}, b_{-2}$ be defined by symmetry with respect to zero. In this case $E_{j}(n, \rho)$, as defined in Lemma 3.1, coincides with the convex hull of $\left\{f(x)^{2} \mu^{2}-i f^{\prime}(x) f(x) \mu+V_{\rho}(x): \mu \in \mathbb{R}, x \in\left(a_{j}, b_{j}\right)\right\}(j= \pm 1, \pm 2)$. We want to prove that, for any $z \in \mathbb{C}$, $\operatorname{dist}\left(z, E_{j}\left(n, \sigma_{n}\right)\right) \rightarrow \infty$ as $n \rightarrow \infty$ if $\sigma_{n}$ tends to zero sufficiently fast. The idea is to show that if $x \in\left(a_{j}, b_{j}\right), \mu \in \mathbb{R}$, then $f(x)^{2} \mu^{2}-i f^{\prime}(x) f(x) \mu+V_{\sigma_{n}}(x)$ is contained in some angular sector of amplitude strictly less than $\pi(j= \pm 1, \pm 2, n$ large): thus the divergence $\lim \left|V_{\sigma_{n}}(x)\right|=+\infty$ will be preserved under convex combinations restricted to $x \in\left(a_{j}\left(n, \sigma_{n}\right), b_{j}\left(n, \sigma_{n}\right)\right)$ and, as a consequence, $\lim \operatorname{dist}\left(z, E_{j}\left(n, \sigma_{n}\right)\right)=+\infty$. Therefore the proof is reduced to the following $n$ statements:

a) $\lim \left|V_{\rho}(x)\right|=+\infty$ uniformly for $0<\rho<\rho_{0}$

b) for suitable $\rho_{n} \rightarrow 0$ let $\sigma_{n}=O\left(\rho_{n}\right)$ : then

$\left.\mathrm{b}_{1}\right) \forall \varepsilon^{\prime}>0, \quad \exists \bar{n}: \forall n>\bar{n}, \quad-\pi+4 \bar{\eta} \leqq \arg V_{\sigma_{n}}(x) \leqq-\pi+4 \beta / 3+2 \bar{\eta}+\varepsilon^{\prime}, \quad \forall x \in$ $(-\infty,-n)$

$\left.\mathrm{b}_{2}\right) \exists \varepsilon^{\prime}>0, \quad \exists \bar{n}: \forall n>\bar{n}, \quad-\pi+4 \beta / 3-2 \bar{\eta} \leqq \arg V_{\sigma_{n}}(x) \leqq 4 \beta / 3-2 \bar{\eta}-\varepsilon^{\prime} \forall x \in$ $\left(n, 2 n+1 / \sigma_{n}\right)$

$\left.\mathrm{b}_{3}\right) \forall \varepsilon^{\prime}>0, \exists \bar{n}: \forall n>\bar{n}, 0 \leqq \arg V_{\sigma_{n}}(x) \leqq \pi-4 \bar{\eta}+\varepsilon^{\prime}, \forall x \in\left(n+1 / \sigma_{n},+\infty\right)$.

To prove part a) it is enough to find a uniform positive lower bound for $\left|e^{2 i \beta / 3}-\rho x e^{i \eta(x)}\right|^{2}=1+\rho^{2} x^{2}-2 \rho x \cos (2 \beta / 3-\eta(x))$. Since $\eta(x)= \pm \bar{\eta}$ for $|x| \geqq 3$, with $\bar{\eta}$ small with respect to $\varepsilon, \beta \in[\varepsilon, 3 \pi / 4]$, we have $\cos (2 \beta / 3-\eta(x))<c<1$, which proves the lower estimate. Part $b_{1}$ ) is immediately suggested by the phases of $-\xi(x)^{4}$ and $-e^{4 i \beta / 3} \xi(x)^{2}$ for $x<-n$. For part $b_{2}$ ) suppose $\beta \in[\varepsilon, 3 \pi / 8]$ (in complete analogy one can treat other $\beta^{\prime} s$ ). It is enough to see that

$$
\begin{gathered}
-\pi+4 \beta / 3-2 \bar{\eta} \leqq \arg V_{\sigma_{n}}(x) \leqq \pi / 2 \\
\exists \varepsilon^{\prime}>0, \exists \bar{n}: \forall n>\bar{n}, \operatorname{Im} V_{\sigma_{n}}(x) \leqq \tan \left(4 \beta / 3-2 \bar{\eta}-\varepsilon^{\prime}\right) \operatorname{Re} V_{\sigma_{n}}(x)
\end{gathered}
$$

are both valid $\forall x \in\left(n, 2 n+1 / \sigma_{n}\right)$. Now $(3.17 a)$ follows from $\operatorname{Im} V_{\sigma_{n}}(x) \leqq \tan (4 \beta / 3-$ $2 \bar{\eta}) \operatorname{Re} V_{\sigma_{n}}(x)$. This inequality in turn reduces to $\sigma_{n} x \leqq L$, where $L=(2 \tan (4 \beta / 3-$ $2 \bar{\eta}) \cos (2 \beta / 3-3 \bar{\eta})-2 \sin (2 \beta / 3-3 \bar{\eta}))(\tan (4 \beta / 3-2 \bar{\eta}) \cos 4 \bar{\eta}+\sin 4 \bar{\eta})^{-1}$. So we have $2 n+1 / \sigma_{n} \leqq L / \sigma_{n}$, for $\sigma_{n} \rightarrow 0$ sufficiently fast, only if $L>1$, which is true by direct inspection. Equation (3.17b) is equivalent to an inequality which is a fortiori verified if $\sigma_{n} x \leqq M\left(\varepsilon^{\prime}\right)$, where $M\left(\varepsilon^{\prime}\right)>1$ for $\varepsilon^{\prime}$ small: so, if $x \in\left(n, 2 n+1 / \sigma_{n}\right)$, then $(3.17 b)$ holds. Similarly one can show part $b_{3}$ ) and the proposition is thus proved.

These results and the analogous ones that we can obtain for $H^{(+)}(g)$ can be summarized as follows.

Theorem 3.5. Every eigenvalue of the harmonic oscillator $p^{2}+x^{2}$ is stable with respect to the families $H^{(+)}(g), H^{(-)}(g)$ defined by (3.9) as $g \rightarrow 0$, if $\mid \arg (g) \pm$ $\pi / 2 \mid<3 \pi / 4$ respectively. In particular, for any $\varepsilon>0$ the stability with respect to both $H^{(+)}(g)$ and $H^{(-)}(g)$ is uniform for $\arg (g) \in[-\pi / 4+\varepsilon, \pi / 4-\varepsilon]$.

In the following section we shall use the solutions $u_{j}(x)=u_{j}(x, g, z)$ of the equation $\left[-d^{2} / d x^{2}+x^{2}(1-g x)^{2}\right] u=z u$ exponentially decaying as $|x| \rightarrow \infty$ in any direction of the sector $S_{j}(g)=\{x \in \mathbb{C}:|\arg x+\arg (g) / 3-\pi j / 3|<\pi / 6\}, \quad j=$ 
$-2,-1, \ldots, 3$. Such solutions are determined up to constant factors, which are taken so that $u_{j}(x, g, z)=\overline{u_{-j}(\bar{x}, \bar{g}, \bar{z})}$. We are interested in the behaviour, as $g \rightarrow 0+$, of the ratio

$$
k(g, z)=\left(W_{0,1}(g, z) W_{3,-1}(g, z)\right)\left(W_{0,-1}(g, z) W_{3,1}(g, z)\right)^{-1},
$$

where $W_{i, j}(g, z)$ is the Wronskian of the solutions $u_{i}, u_{j}$ (notice that $k(g, z)$ does not depend on the choice of the above mentioned constant factors). Similarly let $\tilde{u}_{j}(x, z)$ denote the analogous solutions of the equation $\left[-d^{2} / d x^{2}+x^{2}\right] u=z u$ decaying as $|x| \rightarrow \infty$ in the sectors $\widetilde{S}_{j}=\{x:|\arg x-\pi j / 2|<\pi / 4\}(j=-1, \ldots, 2)$, and let $\tilde{W}_{i, j}(0, z)$ denote the Wronskian of the solutions $\tilde{u}_{i}, \tilde{u}_{j}$. Then we are able to prove the following convergence.

Theorem 3.6. Let $g>0$ and let $z \in \mathbb{C} \backslash \sigma_{p}(H(0))$. Then the function $k(g, z)$ defined by (3.18) satisfies

$$
k(g, z) \rightarrow k(0, z) \equiv\left(\tilde{W}_{0,1}(0, z) \tilde{W}_{2,-1}(0, z)\right)\left(\tilde{W}_{0,-1}(0, z) \tilde{W}_{2,1}(0, z)\right)^{-1}=e^{i(z+1) \pi},
$$

as $g \rightarrow 0$, uniformly on compact sets in $z$.

The proof of Theorem 3.6 comes down to operator techniques the following way:

(A) Expression (3.18) can be written in terms of Green functions:

$$
k(g, z)=\left(G_{0,-1}\left(x_{g}, x_{g} ; g, z\right) G_{3,1}\left(x_{g}, x_{g} ; g, z\right)\right)\left(G_{0,1}\left(x_{g}, x_{g} ; g, z\right) G_{3,-1}\left(x_{g}, x_{g} ; g, z\right)\right)^{-1} \text {. }
$$

Here $G_{i, j}(x, y ; g, z)$ denotes the (formal) Green function associated with the solutions $u_{i}, u_{j}$. Similarly one can define $\widetilde{G}_{i, j}$ and write $k(0, z)$ in terms of $\widetilde{G}_{i, j}$. The point $x_{g}$ is arbitrary, except for the fact that $G_{i, j}\left(x_{g}, x_{g} ; g, z\right) \neq 0$ for any $i$ and $j$.

(B) We consider the closed operator

$$
K_{0,1}(g)=f(x)^{2} p^{2}-i f^{\prime}(x) f(x) p+\xi(x)^{2}(1+g \xi(x))^{2},
$$

with $C_{0}^{\infty}(\mathbb{R})$ as a core. Here $g \geqq 0, \xi(x)=x e^{i \eta(x)}, f(x)=\left(\xi^{\prime}(x)\right)^{-1}$ and $\eta(x)$ is a non-decreasing function such that: $\eta \in C^{\infty}(\mathbb{R}), \eta(x)=-\pi / 2-\bar{\eta}$ for $x \leqq-1(\bar{\eta}>0$ and small), $\eta(x)=0$ for $x \geqq 1$. Similarly we consider the closed operator $K_{3,1}(g)$, having $C_{0}^{\infty}(\mathbb{R})$ as a core, defined by

$$
K_{3,1}(g)=h(x)^{2} p^{2}-i h^{\prime}(x) h(x) p+\zeta(x)^{2}(1+g \zeta(x))^{2} .
$$

Here $g>0, \zeta(x)=x e^{i \vartheta(x)}, h(x)=\left(\zeta^{\prime}(x)\right)^{-1}$ and $\vartheta(x)$ is a non-decreasing function such that $\vartheta \in C^{\infty}(\mathbb{R}), \vartheta(x)=0$ for $x \leqq 2$ and $\vartheta(x)=\bar{\eta}+\pi / 4$ for $x \geqq 3(\bar{\eta}>0$ and small). When $g=0$ we denote $K_{2,1}(0)$ the operator defined this way.

(C) The functions $u_{0}(x+1 / g ; g, z)$ and $u_{1}(x+1 / g ; g, z)$ are fundamental solutions of the equation $K_{0,1}(g) v(x)=z v(x)$ in the interval $(1,+\infty)$. Indeed such equation reduces to $-u^{\prime \prime}(x)+x^{2}(1-g x)^{2} u(x)=z u(x)$ (with $u(x)=v(x-1 / g)$ ) for $x>1$ and, taking into account the deformation which defines $K_{0,1}(g), u_{0}, u_{1}$ are $L^{2}$ at $+\infty,-\infty$ respectively. Similarly $u_{3}(x+1 / g ; g, z)$ and $u_{1}(x+1 / g ; g, z)$ are fundamental solutions of the equation $K_{3,1}(g) v(x)=z v(x)$ in the interval $(-\infty, 2)$. Now, let $1<x_{0}<2$ : by these remarks the Green functions for $K_{0,1}(g)$ and $K_{3,1}(g)$ can be constructed so that, when calculated in $\left(x_{0}, x_{0}\right)$, they coincide with $G_{0,1}\left(x_{0}+\right.$ $\left.1 / g, x_{0}+1 / g\right)$ and $G_{3,1}\left(x_{0}+1 / g, x_{0}+1 / g\right)$ respectively. 
(D) Take $x_{g}=x_{0}+g^{-1}$ in (3.20), for some suitable $x_{0} \in(1,2)$ and consider the Green functions $G_{i, j}^{*}$ of the operators $K_{i, j}(g)$ satisfying the property just quoted in (C) (a symmetric definition and an analogous discussion can be given for the operators $K_{0,-1}(g)$ and $\left.K_{3,-1}(g)\right)$. Then:

$$
k(g, z)=\left(G_{0,-1}^{*}\left(x_{0}, x_{0} ; g, z\right) G_{3,1}^{*}\left(x_{0}, x_{0} ; g, z\right)\right)^{-1}\left(G_{0,1}^{*}\left(x_{0}, x_{0} ; g, z\right) G_{3,-1}^{*}\left(x_{0}, x_{0} ; g, z\right)\right)^{-1} \text {. }
$$

We have the analogous representation of $k(0, z)$ in terms of Green functions $\tilde{G}_{i, j}^{*}$ associated with the operators $K_{i, j}(0)$ defined in (B) and constructed so that $\tilde{G}_{i, j}^{*}\left(x_{0}, x_{0} ; z\right)=\widetilde{G}_{i, j}\left(x_{0}, x_{0} ; z\right)$ for $x_{0} \in(1,2)$.

As a consequence, Theorem 3.6 is proved if we can show that

$$
\begin{array}{lll}
K_{0,1}(g) \rightarrow K_{0,1}(0) & \text { as } & g \rightarrow 0, \\
K_{3,1}(g) \rightarrow K_{2,1}(0) & \text { as } & g \rightarrow 0,
\end{array}
$$

in the strong resolvent sense. If (3.24) is verified, choosing $x_{0} \in(1,2)$ so that $\widetilde{G}_{i, j}\left(x_{0}, x_{0} ; z\right) \neq 0$, we have $G_{i, j}\left(x_{0}, x_{0} ; g, z\right) \neq 0$ for $g$ sufficiently small, the right-hand side of (3.20) is well defined and (3.19) follows.

To prove (3.24) we need the following propositions.

Proposition 3.7. The set $\Delta \equiv\left\{z \in \mathbb{C}: z \notin \sigma_{p}\left(K_{0,1}(g)\right)\right.$ and $\left(K_{0,1}(g)-z\right)^{-1}$ is uniformly bounded for $g \geqq 0\}$ coincides with $\mathbb{C}$.

Proof. An application of Lemma 3.1. To prove the needed estimate (3.1) it is enough to consider $e^{i \beta} K_{0,1}(g)$ (with $\beta=-\pi+3 \bar{\eta} / 2$ ) and to show the existence of a real-valued function $\gamma(x)$ such that:

$$
\operatorname{Re} e^{-i \gamma(x)} e^{i \beta} K_{0,1}(g) \geqq c_{1} p^{2}-c_{2}
$$

(as quadratic forms on $C_{0}^{\infty}(\mathbb{R})$ ) for $c_{1}, c_{2}>0$ independent of $g$. Choosing a non-increasing $C^{\infty}$-function $\gamma(x)$ such that $\gamma(x)=(\pi-\bar{\eta}) / 2$ for $x \leqq-1$ and $\gamma(x)=$ $(-\pi+\bar{\eta}) / 2$ for $x \geqq 1$ and taking into account the behaviour of $\xi(x)$, one can show (3.25) by the same arguments used in the proof of Proposition 3.3. As for the second group of hypotheses of Lemma 3.1, the situation is simplified with respect to the proof of Proposition 3.4. Indeed we can simply take $\left(a_{1}, b_{1}\right)=(n,+\infty)$, $\left(a_{-1}, b_{-1}\right),(-\infty,-n)$. It is easy to verify that $\left\{\left\langle u, e^{i \beta} K_{0,1}(g) u\right\rangle: u \in C_{0}^{\infty}(\mathbb{R}),\|u\|=1\right.$, supp $u \subset(-\infty,-n)\}$ is contained in a sector of amplitude strictly less than $\pi$ uniformly for small $g$, so that $\operatorname{dist}\left(z, E_{-1}(n, g)\right) \rightarrow \infty$ as $n \rightarrow \infty, z \in \mathbb{C}, g$ small. Since the same result holds for $E_{1}(n, g)$, Lemma 3.1 applies for all $z \notin \sigma_{p}\left(K_{0,1}(0)\right)$. This spectrum, in turn, is empty because the solutions $u_{0}(x, z)$ and $u_{1}(x, z)$ (as defined above) are linearly independent for all $z$ (see [12], par. 21) and the Green function associated with $K_{0,1}(0)$ can be constructed by means of solutions coinciding with $\tilde{u}_{0}, \tilde{u}_{1}$ for $x \geqq 1$. Thus Lemma 3.1 applies for any $z \in \mathbb{C}$ and the proposition is proved.

To prove an analogous result for $K_{3,1}(g)$, it is convenient to define $H_{3,1}(g)=$ $T_{-1 / g} K_{3,1}(g) T_{1 / g}$, where $T_{-1 / g} \phi(x)=\phi(x-1 / g) . K_{3,1}(g)$ and $H_{3,1}(g)$ are unitarily equivalent and have the same spectrum, but the formal limits as $g \rightarrow 0+$ are $K_{2,1}(0)$ and $K_{2,0}(0)$ respectively:

$$
K_{2,1}(0)=h(x)^{2} p^{2}-i h^{\prime}(x) h(x) p+\zeta(x)^{2},
$$




$$
K_{2,0}(0)=p^{2}+x^{2} \text {. }
$$

If we consider the closure of the differential expressions (3.26) on $C_{0}^{\infty}(\mathbb{R}), K_{2,0}(0)$ is the usual harmonic oscillator, while $K_{2,1}(0)$ has empty spectrum (indeed the solutions $\tilde{u}_{2}(x, z), \tilde{u}_{1}(x, z)$ are linearly independent for all $z$, and the Green function associated with $K_{2,1}(0)$ can be constructed by means of solutions coinciding with $\tilde{u}_{2}, \tilde{u}_{1}$ for $x \leqq 2$ ).

Proposition 3.8. The operators $K_{3,1}(g)$ and $H_{3,1}(g)$ satisfy (3.1) uniformly for $g \geqq 0$.

Proof. Let $\gamma$ be a non-increasing $C^{\infty}$-function such that $\gamma(x)=-\pi / 4$ for $x \leqq 2$ and $\gamma(x)=-\pi$ for $x \geqq 3$. Proceeding as in Proposition 3.3 the required estimate is proved for $e^{i \gamma(x)} K_{3,1}(g)$, and hence for $K_{3,1}(g)$ and $H_{3,1}(g)$.

Let $\chi \in C_{0}^{\infty}(\mathbb{R}), \chi(x)=1$ for $|x| \leqq 1, \chi(x)=0$ for $|x| \geqq 2,0 \leqq \chi(x) \leqq 1$, and let $\chi_{n}(x)=\chi(x / n), n \in \mathbb{N}$. Let us define, for $g$ sufficiently small with respect to $1 / n$,

$$
\begin{aligned}
\chi_{n}^{g}(x) & = \begin{cases}\chi(x / n), & x \geqq-1 / 2 g \\
\chi((x+1 / g) / n)=T_{1 / g} \chi_{n}(x), & x \leqq-1 / 2 g\end{cases} \\
M_{n}(g) & =1-\chi_{n}^{g} .
\end{aligned}
$$

\section{Proposition 3.9.}

a) $M_{n}(g)$ is uniformly bounded in $n$ and $g$.

b) If $g_{n} \rightarrow 0+$ and $u_{m} \in D\left(K_{3,1}\left(g_{m}\right)\right), \quad\left\|u_{m}\right\| \rightarrow 1, u_{m} \stackrel{w}{\longrightarrow} 0, v_{m} \equiv T_{-1 / g_{m}} u_{m} \stackrel{w}{\longrightarrow} 0$ and $\left\|K_{3,1}\left(g_{m}\right) u_{m}\right\| \leqq C$, for some $C>0, \forall m$, then

$$
\lim _{m}\left\|M_{n}\left(g_{m}\right) u_{m}\right\|=1, \quad \forall n .
$$

c) $\left\|\left[M_{n}(g), K_{3,1}(g)\right] u\right\| \leqq c n^{-1}\left(\left\|K_{3,1}(g) u\right\|+\|u\|\right)$, uniformly with respect to $g \geqq 0$ and $n \in \mathbb{N}$.

Proof. The first assertion is trivial. To prove b) notice that, for $g_{m}$ sufficiently small,

$$
\left\|\chi_{n}^{g_{m}} u_{m}\right\|^{2}=\left\|\chi_{n} u_{m}\right\|^{2}+\left\|\chi_{n} v_{m}\right\|^{2} \rightarrow 0 \quad \text { as } \quad m \rightarrow \infty .
$$

Indeed $\chi_{n}\left(1+p^{2}\right)^{-1 / 2}$ is a compact operator independent of $m$, while $\left(1+p^{2}\right)^{1 / 2} v_{m} \stackrel{w}{\longrightarrow} 0$ by Proposition 3.8. This proves that $\left\|M_{n}\left(g_{m}\right) u_{m}\right\| \rightarrow 1$, for all $n$, as $m \rightarrow \infty$. Since the derivatives of $\chi_{n}^{g}$ are bounded independently of $(g, c)$ is proved using Proposition 3.8 .

Definition. Let $\lambda \in \mathbb{C}$ and let

$$
d_{n}^{ \pm}(\lambda, g)=\inf \left\{\left\|\left(\lambda-K_{3,1}(g)\right) M_{n}^{ \pm}(g) u\right\|: \quad u \in D\left(K_{3,1}(g)\right), \quad\left\|M_{n}^{ \pm}(g) u\right\|=1\right\},
$$

where $\quad M_{n}^{+}(g)(x)=M_{n}(g)(x) \quad$ if $\quad x \geqq 0, M_{n}^{-}(g)(x)=M_{n}(g)(x) \quad$ if $\quad x<0, \quad$ and $M_{n}^{ \pm}(g)(x)=0$ otherwise.

\section{Proposition 3.10.}

(i) If $\left\{g_{m}, u_{m}\right\}$ satisfies b) of Proposition 3.9, then for any $n \in \mathbb{N}$ we have limsup $\left\|M_{n}^{+}\left(g_{m}\right) u_{m}\right\| \geqq 1 / 2$ or limsup $\left\|M_{n}^{-}\left(g_{m}\right) u_{m}\right\| \geqq 1 / 2$. 
(ii) Point c) of Proposition 3.9 holds both if $M_{n}\left(g_{m}\right)$ is replaced by $M_{n}^{+}\left(g_{m}\right)$ and by $M_{n}^{-}\left(g_{m}\right)$.

(iii) $\forall \lambda \in \mathbb{C}, \lim _{\substack{n \rightarrow \infty \\ g \rightarrow 0+}} d_{n}^{ \pm}(\lambda, g)=+\infty$.

Proof. The first two assertions are easily verified. We have $\lim _{x \rightarrow+\infty}|\zeta(x)(1+g \zeta(x))|^{2}=$ $+\infty$ uniformly in $g \geqq 0$. Moreover the values taken on by the potential and by $h(x)^{2} p^{2}-i h^{\prime}(x) h(x) p$ remain in some sector whose amplitude is less than $\pi$ uniformly in $g$. Hence $d_{n}^{+}(\lambda, g) \rightarrow+\infty$. For $d_{n}^{-}(\lambda, g)$ we have to consider $K_{3,1}(g)$ for $x<-n,\left|x+g^{-1}\right|>n$, where $K_{3,1}(g)=p^{2}+x^{2}(1+g x)^{2}$. Thus the proposition is proved.

Proposition 3.11. Let $\lambda \in \mathbb{C} \backslash \sigma(H(0))$. Then $\lambda \in \Delta$ unless there exist $g_{m} \rightarrow 0+, u_{m} \in D$ $\left(K_{3,1}\left(g_{m}\right)\right), \quad\left\|u_{m}\right\| \rightarrow 1, \quad u_{m} \stackrel{w}{\longrightarrow} 0, \quad v_{m}=T_{-1 / g_{m}} u_{m} \stackrel{w}{\longrightarrow} 0, \quad\left\|\left(\lambda-K_{3,1}\left(g_{m}\right)\right) u_{m}\right\| \rightarrow 0$, $\left\|\left(\lambda-H_{3,1}\left(g_{m}\right)\right) v_{m}\right\| \rightarrow 0$.

Proof. Proceeding as in Lemma 5.1 of [15], we have only to verify that $v_{m} \stackrel{w}{\longrightarrow} 0$. Since $\left\|\left(\lambda-K_{3,1}\left(g_{m}\right)\right) u_{m}\right\| \rightarrow 0,\left\|\left(\lambda-H_{3,1}\left(g_{m}\right)\right) v_{m}\right\| \rightarrow 0$. Now, passing to a subsequence if necessary, $v_{m} \stackrel{w}{\longrightarrow} v$ so that, for any $\psi \in C_{0}^{\infty}(\mathbb{R})$,

$$
0=\lim _{m}\left\langle\psi,\left(\lambda-H_{3,1}\left(g_{m}\right)\right) v_{m}\right\rangle=\lim _{m}\left\langle\left(\lambda-H_{3,1}\left(g_{m}\right)\right)^{*} \psi, v_{m}\right\rangle=\left\langle(\lambda-H(0))^{*} \psi, v\right\rangle,
$$

which implies $v=0$, and the assertion is proved.

Proposition 3.12. The resolvents $\left(\lambda-K_{3,1}(g)\right)^{-1}$ are uniformly bounded as $g \rightarrow 0+$ for $\lambda \in \mathbb{C} \backslash \sigma(H(0))$.

Proof. If $\lambda \in \mathbb{C} \backslash \sigma(H(0))$ and $\lambda \notin \Delta$, then there are two sequences $\left\{g_{n}, u_{n}\right\}$ satisfying the properties stated in Proposition 3.11. By Proposition 3.9 the same properties are satisfied by $\left\{g_{m(n)}, M_{n}\left(g_{m(n)}\right) u_{m(n)}\right\}$ as $n \rightarrow \infty$. Indeed, we only need to check the weak convergence to zero of $M_{n}\left(g_{m}\right) u_{m}$ and of $T_{-1 / g_{m}} M_{n}\left(g_{m}\right) T_{1 / g_{m}} T_{-1 / g_{m}} u_{m}$ (with $m=m(n))$ as $n \rightarrow \infty$. Such weak convergence occurs since both the operators $M_{n}(g)$ and $T_{-1 / g} M_{n}(g) T_{1 / g}$ strongly tend to zero uniformly in $g$. Then we know from (i)-(ii) of Proposition 3.10 that the same properties are satisfied by both $\left\{g_{m}, M_{n}^{+}\left(g_{m}\right) u_{m}\right\}$ and $\left\{g_{m}, M_{n}^{-}\left(g_{m}\right) u_{m}\right\}, m=m(n)$. Therefore (iii) of Proposition 3.10 is contradicted and $\lambda \in \Delta$.

Now for the model $H_{1}(g)=p^{2}+x^{2}(1-g x)^{2}+2 g x-1$, one can prove the following statements in full analogy with the preceding theorems.

\section{Theorem 3.13.}

(i) The ground state eigenvalue of $p^{2}+x^{2}-1 \equiv H(0)-1$ is stable with respect to the family $H_{1}(g)=p^{2}+x^{2}(1-g x)^{2}+2 g x-1$ (defined so as to have $C_{0}^{\infty}(\mathbb{R})$ as a core).

(ii) The eigenvalues of $H(0)-1=p^{2}+x^{2}-1$ are stable with respect to the two families of "resonance" operators $H_{1}^{( \pm)}(g)$ defined in analogy with (3.9).

(iii) If $k(g, z)$ denotes the ratio (3.18) constructed by means of solutions of $H_{1}(g) u=z u(g>0)$, then $k(g, z) \rightarrow k(0, z-1) \equiv e^{i z \pi}$ as $g \rightarrow 0$. 


\section{The Method}

a) The Resolvent Formula. A crucial point of the method consists in the expansion of some matrix elements of the resolvent in terms of matrix elements of the two Hermitian conjugate resolvents for the "resonances" (see (4.6) below). This result applies unchanged to case (I) and case (II). Now let $H(g)=p^{2}+V(g)=H_{k}(g)$, $k=1,2$, for $g \in \mathbb{C}, g \neq 0, \vartheta=\arg (g)$. Following Sibuya [12] we introduce the fundamental solutions $u_{j}=u_{j}(g, z)$ of the formal Schrödinger equation $H(g) u=z u$, each vanishing for $|x| \rightarrow \infty$ in any direction in the sector $S_{j}(g)=\{x \in \mathbb{C}: \mid \arg (x)+$ $\vartheta / 3-\pi j / 3 \mid<\pi / 6\},-2 \leqq j \leqq 3$, and completely determined up to a constant factor, here taken in such a way that $u_{j}(g, z)=\overline{u_{-j}(\bar{g}, \bar{z})}$ for $x \in \mathbb{R}$. This is possible because the equation is real for $x, g, z$ real and the conditions are complex conjugate. Let $W_{i, j}=W_{i, j}(g, z)=u_{i}(x) u_{j}^{\prime}(x)-u_{i}^{\prime}(x) u_{j}(x), \forall x \in \mathbb{R}$, be the Wronskian of two solutions, and suppose $W_{1,-1}=-W_{-1,1} \neq 0$. In this case $\left\{u_{1}, u_{-1}\right\}$ is a basis for the space of the solutions of the Schrödinger equation, and we have:

$$
u_{0}=c_{+} u_{1}+c_{-} u_{-1},
$$

where $c_{ \pm}=W_{0, \mp 1} / W_{ \pm 1, \mp 1}$ are the Stokes multipliers of $u_{0}$ with respect to $\left\{u_{1}, u_{-1}\right\}$ (see [12] page 83).

Let $W_{j, k} \neq 0$ and define the two-index "kernels":

$$
G_{j, k}(x, y)=\left\{\begin{array}{ll}
u_{j}(x) u_{k}(y) / W_{j, k} & x \leqq y \\
u_{j}(y) u_{k}(x) / W_{j, k} & y \leqq x
\end{array} .\right.
$$

In particular, if $W_{0,3}, W_{3, \pm 1}$ are different from zero, using (4.1) and (4.2), we have the relation:

$$
\begin{aligned}
G_{3,0}(x, y) & =d_{+} G_{3,1}(x, y)+d_{-} G_{3,-1}(x, y) \\
& =\frac{1}{2}(1+i h) G_{3,1}(x, y)+\frac{1}{2}(1-i h) G_{3,-1}(x, y),
\end{aligned}
$$

where $\quad d_{ \pm}=c_{ \pm} W_{3, \pm 1} / W_{3,0}=W_{0, \mp 1} W_{3, \pm 1} / W_{ \pm 1, \mp 1} W_{3,0}=\left(1-k^{ \pm 1}\right)^{-1}, k=W_{0,1} W_{3,-1} /$ $W_{0,-1} W_{3,1}$, and $h=h(g, z)=\overline{h(\bar{g}, \bar{z})}=-i(1+k) /(1-k)$. We set $G(x, y)=G_{3,0}(x, y)$, since it is the kernel of the resolvent $R=R(g, z)=(H(g)-z)^{-1}$. Now we state the main result of this paragraph a):

Lemma 4.1. Let $R=R(g, z)=(H(g)-z)^{-1}$ for $z \notin \sigma(H(g)), g \neq 0,|\vartheta|<\pi / 4$, and $\phi, \psi \in N_{\pi / 4}$ the class of dilation analytic vectors $[10]: N_{\pi / 4}=\left\{\psi \in L^{2}(\mathbb{R}): \psi_{\alpha}\right.$ is analytically continuable from $\operatorname{Re}(\alpha)=0$ to $|\operatorname{Re}(\alpha)|<\pi / 4$, where $\psi_{\alpha}(x)=\left(U_{\alpha} \psi\right)(x)=$ $\left.e^{i \alpha / 2} \psi\left(e^{i \alpha} x\right)\right\}$, containing the set $\left\{e^{-x^{2} / 2} P(x): P(x)\right.$ is a polynomial $\}$ dense in $L^{2}(\mathbb{R})$. Then

$$
\langle\phi, R \psi\rangle=\frac{1}{2}(1+i h)\left\langle\phi_{-\alpha}, R^{(+)} \psi_{\alpha}\right\rangle+\frac{1}{2}(1-i h)\left\langle\phi_{\alpha^{\prime}}, R^{(-)} \psi_{-\alpha^{\prime}}\right\rangle,
$$

where $R^{( \pm)}=R^{( \pm)}(g, z)=\left(H^{( \pm)}(g)-z\right)^{-1}$, and $H^{(+)}(g)\left(H^{(-)}(g)\right)$, defined in (3.38) as the "resonance operator" (see also [6]), is formally given by $H^{(+)}(g)=U_{\alpha} H(g) U_{-\alpha}$ $\left(H^{(-)}(g)=U_{-\alpha^{\prime}} H(g) U_{\alpha^{\prime}}=H^{(+) *}(\bar{g})\right), \quad$ where $\quad \alpha=\alpha(g)=\pi / 6-\vartheta / 3\left(\alpha^{\prime}=\alpha(\bar{g})=\right.$ $\pi / 6+\vartheta / 3)$.

Proof. The operator $R^{(+)}$(and similarly $R^{(-)}$) is directly defined through the Green 
function $G^{(+)}(x, y)$ as integral kernel [7], where:

$$
G^{(+)}(x, y)=G_{3,1, \alpha}(x, y)=\left\{\begin{array}{ll}
u_{3, \alpha}(x) u_{1, \alpha}(y) / e^{-2 i \alpha} W\left(u_{3, \alpha}, u_{1, \alpha}\right) & x \leqq y \\
u_{3, \alpha}(y) u_{1, \alpha}(x) / e^{-2 i \alpha} W\left(u_{3, \alpha}, u_{1, \alpha}\right) & y \leqq x
\end{array},\right.
$$

with $u_{j, \alpha}=U_{\alpha} u_{j}$, and $G_{3,1, \alpha}(x, y)=e^{i \alpha} G_{3,1}\left(e^{i \alpha} x, e^{i \alpha} y\right)$. In order to prove (4.4) we consider for $g \neq 0,|\arg (g)|<\pi / 4$, the class $D_{g}$ dense in $L^{2}(\mathbb{R})$ given by: $D_{g}=$ $\left\{\phi_{g}: \phi_{g}(x)=P(x) \exp \left(-g\left(x^{2}+1\right)^{3 / 2}-x^{2}\right)\right.$, for some polynomial $\left.P(x)\right\}$. Let $\phi_{g}, \psi_{g} \in D_{g}$; then we have

$$
\begin{aligned}
\left\langle\phi_{\bar{g}}, R \psi_{g}\right\rangle= & \int_{\mathbb{R}^{2}} \overline{\phi_{\bar{g}}(x)} G(x, y) \psi_{g}(y) d x d y=\frac{1}{2}(1+i h) \int_{\mathbb{R}^{2}} \overline{\phi_{\bar{g}}(x)} G_{3,1}(x, y) \psi_{g}(y) d x d y \\
& +\frac{1}{2}(1-i h) \int_{\mathbb{R}^{2}} \overline{\phi_{\bar{g}}(x)} G_{3,-1}(x, y) \psi_{g}(y) d x d y \\
= & \frac{1}{2}(1+i h) \int_{\mathbb{R}^{2}} \overline{\phi_{\bar{g},-\alpha}(x)} G_{3,1, \alpha}(x, y) \psi_{g, \alpha}(y) d x d y \\
& +\frac{1}{2}(1-i h) \int_{\mathbb{R}^{2}} \overline{\phi_{\bar{g}, \alpha^{\prime}}(x)} G_{3,-1, \alpha^{\prime}}(x, y) \psi_{g,-\alpha^{\prime}}(y) d x d y \\
= & \frac{1}{2}(1+i h)\left\langle\phi_{\bar{g},-\alpha}, R^{(+)} \psi_{g, \alpha}\right\rangle+\frac{1}{2}(1-i h)\left\langle\phi_{\bar{g}, \alpha^{\prime}}, R^{(-)} \psi_{g,-\alpha^{\prime}}\right\rangle,
\end{aligned}
$$

where we have performed a change of path and of variable: $(x, y) \rightarrow e^{i \alpha}(x, y)$, $(x, y) \rightarrow e^{-i \alpha^{\prime}}(x, y)$ in the first and the second integral respectively, justified by the rapid decay of the integrand in the intermediate directions at infinity. In fact [12] we have $u_{j}(x) \sim c_{j} x^{v} \exp \left( \pm\left(g x^{3} / 3-x^{2} / 2\right)\right),-2 \leqq j \leqq 3$, and $\phi_{g}(x) \sim$ $c_{n}^{\prime} x^{n} \exp \left(-g|x|^{3}-x^{2}-\frac{3}{2} g|x|\right)$ as $|x| \rightarrow \infty$ in $S_{0}(g)$. The proof is completed considering the density of $D_{g}$ for any $g,|\arg (g)|\langle\pi / 4$, and the continuity of $\langle\phi, R \psi\rangle$ in the vectors $\phi, \psi$.

b I) The Perturbation Formula for Case (I). In case (I) we can apply (see Theorem 3.13) the perturbation theory for an isolated stable eigenvalue as $g \rightarrow 0^{+}$, so that for small positive $g$, we have

$$
E(g)=N(g) / D(g),
$$

where $N(g)=(2 \pi i)^{-1} \oint_{\Gamma} z\left\langle\psi_{1}, R(g, z) \psi_{2}\right\rangle d z, D(g)=(2 \pi i)^{-1} \oint_{\Gamma}\left\langle\psi_{1}, R(g, z) \psi_{2}\right\rangle d z \neq 0$, and $\Gamma$ is a closed path encircling the origin (for instance the circle $|z|=1$ ). $\psi_{1}, \psi_{2}$, possibly dependent on $g$, should belong to $N_{\pi / 4}$ for our purpose. Actually, in order to have the most explicit results with the use of (4.4), it is convenient to extend (4.7) to formal vectors $\psi_{1}(x)=\psi_{2}(x)=\psi(g, x)=g \exp \left(g x^{3} / 3-\frac{1}{2} x^{2}\right)$. We notice that $\psi(\gamma, x)=\gamma \exp \left(i|\gamma| x^{3} / 3-\frac{1}{2} x^{2} e^{2 i \alpha}\right)$, where $\alpha=\pi / 6-\vartheta / 3, \vartheta=\arg (\gamma), \quad-\pi / 4<\vartheta<$ $5 \pi / 4$, is an eigenvector of $H^{+}(\gamma)$ with eigenvalue $\lambda=0$, the real "resonance" associated with the ground state. This choice of vectors is justified by formula (4.4) and the existence, for any given $g$, of a sequence $\left\{\phi_{(n)}\right\}$ belonging $N_{\pi / 4}$ such that $\phi_{(n) \pm \pi / 6} \underset{n \rightarrow 0}{\longrightarrow} \psi_{ \pm \pi / 6}(g)$. In (4.4) we can disregard the term containing $R^{(-)}$, since we have $\langle\psi, R \psi\rangle=\operatorname{Re}\left((1+i h)\left\langle\psi_{-\pi / 6}, R^{(+)} \psi_{\pi / 6}\right\rangle\right)$ for positive $g$ and $\psi \in N_{\pi / 4}$. Thus we have

$$
N(g)=F_{1}(g, g), \quad D(g)=F_{0}(g, g)
$$


where $F_{j}(g, \gamma)=\left(\Phi_{j}(g, \gamma)+\overline{\Phi_{j}(g, \bar{\gamma})}\right) / 2$ and

$$
\Phi_{j}(g, \gamma)=(2 \pi i)^{-1} \oint_{\Gamma} z^{j}(1+i h(g, z))\left\langle\psi_{-\alpha}(\bar{\gamma}), R^{(+)}(\gamma, z) \psi_{\alpha}(\gamma)\right\rangle d z
$$

for $-\pi / 4<\arg (\gamma)<5 \pi / 4,|\gamma|$ small and positive. Since $R^{(+)}(\gamma, z) \psi_{\alpha}(\gamma)=-z^{-1} \psi_{\alpha}(\gamma)$ for $z \neq 0$, we have

$$
\Phi_{j}(g, \gamma)=-G\left(\gamma^{2}\right)(2 \pi i)^{-1} \oint_{\Gamma} z^{j-1}(1+i h(g, z)) d z=-G\left(\gamma^{2}\right)\left(\delta_{j, 0}+i f_{j}(g)\right)
$$

where $f_{j}(g)=\operatorname{Res}\left(z^{j} h(g, z), z=0\right)$ and $G\left(\gamma^{2}\right)=\gamma^{2} \int_{-\infty}^{+\infty} \exp \left(2 i|\gamma| x^{3} / 3-e^{2 i \alpha} x^{2}\right) e^{i \alpha} d x$ is a bounded analytic function in any region $\operatorname{Re}\left(\gamma^{-2}\right)>\rho^{-2}, \rho>0$. In fact in such a region we have the estimate

$$
\begin{aligned}
\left|G\left(\gamma^{2}\right)\right| & \leqq|\gamma|^{2} \int_{-\infty}^{+\infty} \exp \left(-x^{2} \cos (2 \alpha)\right) d x=|\gamma|\left(\pi|\gamma|^{2} / \sin (\varepsilon)\right)^{1 / 2} \\
& <|\gamma| \rho(3 \pi \omega(3 \varepsilon) / \omega(\varepsilon))^{1 / 2}<|\gamma| \rho(3 \pi)^{1 / 2}
\end{aligned}
$$

where $\varepsilon=\pi / 2-2 \alpha=2 \vartheta / 3+\pi / 6,0<\varepsilon<\pi / 3$, and $\omega(\alpha)=\sin (\alpha) / \alpha$ is positive and decreasing in $(0, \pi)$. It is also easy to prove that $G\left(\gamma^{2}\right) \sim \sum_{n=1}^{\infty} a_{n} \gamma^{2 n}$ with the following estimate for the remainders:

$$
\left|R_{N}\left(\gamma^{2}\right)\right|=\left|G\left(\gamma^{2}\right)-\sum_{n=1}^{N-1} a_{n} \gamma^{2 n}\right| \leqq C(\bar{\varepsilon})^{N} N !|\gamma|^{2 N}
$$

for $\arg (\gamma) \geqq-\pi / 4+\bar{\varepsilon}, \operatorname{Re}\left(\gamma^{-2}\right)>\rho^{-2}$, where $a_{n}=(2 / 3)^{2 n-2} \Gamma(3 n-5 / 2) /(2 n-2)$ !. Thus we have the following.

Theorem 4.2. Let $E(g)$ be the first eigenvalue of the operator $H_{1}(g)$ of Herbst-Simon [10], $H_{1}(g)=p^{2}+x^{2}(1-g x)^{2}-1+2 g x$. Then

(i) For any $\varepsilon, 0<\varepsilon<\pi / 4$ there exists $c_{\varepsilon}>0$ such that for $0<g<c_{\varepsilon}$ we have $E(g)=N(g) / D(g)$ with $N(g)=F_{1}(g, g), D(g)=F_{0}(g, g)$, and

$$
F_{j}(g, \gamma)=\left(\Phi_{j}(g, \gamma)+\overline{\Phi_{j}(g, \bar{\gamma})}\right) / 2,
$$

where $\Phi_{j}(g, \gamma)$ is the Borel sum of its asymptotic expansion $\Sigma=\sum_{n=0}^{\infty}\left(a_{j, n}+i b_{j, n}(g)\right) \gamma^{2 n}$ in the domains

$$
\Delta_{\varepsilon}^{1}=\left\{\gamma \in \mathbb{C}: \varepsilon<\arg (\gamma)<\pi-\varepsilon,|\gamma|<c_{\varepsilon}\right\}
$$

with analytic continuation to the domains

$$
\Delta_{\varepsilon}^{2}=\left\{\gamma \in \mathbb{C}:-\pi / 4+\varepsilon<\arg (\gamma)<5 \pi / 4-\varepsilon,|\gamma|<c_{\varepsilon}\right\} .
$$

(ii) Fix $\varepsilon=\eta$ and set $c=c_{\eta}$. For any fixed $g, 0<g<c, F_{j}(g, \gamma)$ is analytic in the disc $\operatorname{Re}\left(\gamma^{-2}\right)>c^{-2}$, and it can be decomposed in two terms

$$
F_{j}(g, \gamma)=F_{j}^{R}(\gamma)+\frac{1}{2} i d_{j}^{I}(g, \gamma)
$$

where $F_{j}^{R}(\gamma)=\left(\Phi_{j}^{R}(\gamma)+\Phi_{j}^{R}(\bar{\gamma})\right) / 2, d_{j}^{I}(g, \gamma)=\Phi_{j}^{I}(g, \gamma)-\overline{\Phi_{j}^{I}(g, \bar{\gamma})}$ are the distributional 
Borel sum and the "discontinuity" of the series $\sum_{n=0}^{\infty} a_{j, n} \gamma^{2 n}, \sum_{n=0}^{\infty} b_{j, n}(g) \gamma^{2 n}$ respectively (see $[3,4])$.

(iii) $\Phi_{j}^{R}(\gamma)=-G\left(\gamma^{2}\right) \delta_{j, 0}, \quad \Phi_{j}^{I}(g, \gamma)=-G\left(\gamma^{2}\right) f_{j}(g)$,

where $G\left(\gamma^{2}\right)=\gamma^{2} \int_{-\infty}^{\infty} \exp \left(2 i|\gamma| x^{3} / 3-e^{2 i \alpha} x^{2}\right) e^{i \alpha} d x$ and $f_{j}(g)=\operatorname{Res}\left(z^{i} h(g, z), z=0\right) \rightarrow$ $\underset{g \rightarrow 0^{+}}{\longrightarrow}(2 / \pi) \delta_{j, 1}, \alpha=\pi / 6-\arg (\gamma) / 3$.

Remark. 4.3. Point (i) means that $\Phi_{j}(g, \gamma)$ for fixed $g$ is a function of $\gamma$ which at $\gamma=g>0$ is the analytic continuation of a Borel sum for $\operatorname{Im}(\gamma)>0$. Point (ii) means that $\Phi_{j}(g, \gamma)$ at $\gamma=g>0$ is also an "upper sum" (see $[3,4]$ ) of its asymptotic expansion, but since the series is complex, $F_{j}(g, \gamma)$ at $\gamma=g$ is not the distributional Borel sum of the same expansion. In fact $F_{j}(g, \gamma)$ can be written as a combination of the distributional Borel sum (DBS) of the "real part" of the series and the "discontinuity" uniquely associated with the "imaginary part" of the series by the DBS criterion (see $[3,4]$ ).

Proof of Theorem 4.2. The proof of (i), (ii) follows from Theorem 3.13 and (iii); a direct operator proof of (i) can be given in the same way as in the subsequent Theorem 4.4. In fact $h(g, z) \underset{g \rightarrow 0^{+}}{\longrightarrow} \operatorname{cotan}(\pi z / 2)$ by Theorem 3.13 uniformly in the compact set $\Gamma$, and so $f_{j}(g) \underset{g \rightarrow 0^{+}}{\longrightarrow} \operatorname{Res}\left(z^{j} \operatorname{cotan}(\pi z / 2), z=0\right)=\delta_{j, 1} 2 / \pi$.

b II) The Perturbation Formula for Case II). Because of the symmetry of the operators $H(g)=H_{2}(g)$ with respect to the point $x=1 / 2 g$ we have proved the stability of the eigenvalues of $H(0)$ with respect to the operators $K^{ \pm}(g)$ formally given by

$$
K^{ \pm}(g)=P^{ \pm}(g) H(g) P^{ \pm}(g)=H(g) P^{ \pm}(g)=P^{ \pm}(g) H(g)
$$

as $g \rightarrow 0^{+}$, where $P^{ \pm}(g)=(1 \pm U(g)) / 2,(U(g) f)(x)=f\left(g^{-1}-x\right)$ (see Sect. 2, statements (i), (ii), (iii), where the result is suitably extended to the sector $|\arg (g)|=$ $|\vartheta|<\pi / 4)$. Let $P(g)=P^{ \pm}(g)$ for a fixed choice of + or,$- g>0$, and $\psi(g)=2 P(g) \psi(0)$ where $\psi(0)=\psi_{n}$ is the eigenfunction of $H(0)$ with eigenvalue $E(0)=2 n+1$, for $n=0,1, \ldots,\|\psi(0)\|=1, \psi(0)=\overline{\psi(0)}$. We have (4.7) with $R=R(g, z)=(H(g)-z)^{-1}$ and $\psi_{1}=\psi_{2}=\psi(g)$. In fact the projection operator $P(g)$ in the expression of $\psi(g)$ selects the space of even or odd functions, in such a way that $H(g)$ acts as $K^{+}(g)$ or $K^{-}(g)$ and we have the stability of the perturbation formula (4.7). By (4.15) we have the identity

$$
\langle\psi(g), R(g, z) \psi(g)\rangle=\langle\psi(g), R(g, z) \psi(0)\rangle+\langle\psi(0), R(g, z) \psi(g)\rangle,
$$

where $g>0$. By (4.7), (4.4) and Propositions 3.3, 3.4, we have

where

$$
N(g)=\operatorname{Re}\left(\Phi_{1}(g, g)\right), \quad D(g)=\operatorname{Re}\left(\Phi_{0}(g, g)\right)
$$

$$
\begin{aligned}
\Phi_{j}(g, \gamma)= & (2 \pi i)^{-1} \oint_{\Gamma} z^{j}(1+i h(g, z))\left(\left\langle\psi_{-\alpha}(g), R^{(+)}(\gamma, z) \psi_{\alpha}(0)\right\rangle\right. \\
& +\overline{\left.\left\langle\psi_{\alpha}(g), R^{(-)}(\bar{\gamma}, \bar{z}) \psi_{\alpha}(\theta)\right\rangle\right) d z}
\end{aligned}
$$


$\Gamma$ is a path encircling $E(0)$ at distance $1, \alpha=\pi / 6-\vartheta / 3,-\pi / 4<\vartheta=\arg (\gamma)<5 \pi / 4$, and $|\gamma|, g$ small enough. Thus we are able to prove:

Theorem 4.4. Let $E(0)=2 n+1, n=0,1, \ldots$, be an eigenvalue of $H(0)=H_{2}(0)$ with eigenvector $\psi(0)$. Then

(i) For any $\varepsilon, 0<\varepsilon<\pi / 4, \exists c_{\varepsilon}>0$ such that there exists an isolated eigenvalue $E(g)=E^{ \pm}(g)$ of $K^{ \pm}(g)$ analytic in $\Delta_{\varepsilon}=\left\{g \in \mathbb{C}:|\arg (g)|<(\pi / 4)-\varepsilon,|g|<c_{\varepsilon}\right\}$ with limit $E(0)$ as $g \rightarrow 0$.

(ii) For fixed $\varepsilon$ and $g, 0<g<c_{\varepsilon}$, we have (4.7) with $N(g)=F_{1}(g, g), D(g)=F_{0}(g, g)$ and

$$
F_{j}(g, \gamma)=\left(\Phi_{j}(g, \gamma)+\overline{\Phi_{j}(g, \bar{\gamma})}\right) / 2
$$

where $\Phi_{j}(g, \gamma)$, given by (4.18), is analytic in the domain $\Delta_{\varepsilon}^{2}=\{\gamma \in \mathbb{C}:-(\pi / 4)+$ $\left.\varepsilon<\arg (\gamma)<(5 \pi / 4)-\varepsilon, 0<|\gamma|<c_{\varepsilon}\right\}$ and is the Borel sum of order $\frac{1}{2}[3]$ of its asymptotic expansion $\sum_{k=0}^{\infty}\left(a_{j k}(g)+i b_{j k}(g)\right) \gamma^{k}$ in the domain $\Delta_{\varepsilon}^{1}=\{\gamma \in \mathbb{C}: \varepsilon<\arg (\gamma)<$ $\left.\pi-\varepsilon, 0<|\gamma|<c_{\varepsilon}\right\}$.

(iii) Any $a_{j k}(g)$ is directly computable and we have $a_{j k}(g)=a_{j k}(0)+P_{2 n+3 k}(1 / g)$ $\exp \left(-(2 g)^{-2}\right)$, where $P_{k}$ is a polynomial of degree $k$. We have the same result for $b_{j k}(g)$ in the approximation $h(g, z) \cong \tilde{h}(g, z)=h(0, z)=\operatorname{cotan}((z+1) \pi / 2)$.

Remark 4.5. In this case we are not able to prove that, for fixed $g>0, F_{j}(g, \gamma)$ is a combination of a distributional Borel sum and a "discontinuity" (see $[3,4]$ ). In particular we are not able to prove the analyticity on the $\operatorname{disc} \operatorname{Re}\left(\gamma^{-2}\right)>C^{-2}$. We recall that this result is claimed by Crutchfield [5] by non-rigorous arguments. In any case for $\gamma$ in $\Delta_{\varepsilon}$ we can write

$$
F_{j}(g, \gamma)=F_{j}^{R}(g, \gamma)+\frac{1}{2} i d_{j}^{I}(g, \gamma),
$$

where $F_{j}^{R}(g, \gamma)=\left(\Phi_{j}^{R}(g, \gamma)+\overline{\Phi_{j}^{R}(g, \bar{\gamma})}\right) / 2, d_{j}^{I}(g, \gamma)=\Phi_{j}^{I}(g, \gamma)-\overline{\Phi_{j}^{I}(g, \bar{\gamma})}$ and $\Phi_{j}^{R}(g, \gamma)$, $\Phi_{j}^{I}(g, \gamma)$ are the Borel sums of order $\frac{1}{2}$ of $\sum_{k=0}^{\infty} a_{j k}(g) \gamma^{k}, \sum_{k=0}^{\infty} b_{j k}(g) \gamma^{k}$ respectively in $\Delta_{\varepsilon}^{1}$.

Proof of Theorem 4.4. Point (i) follows from the stability results of Sect. 2, taking into account Remark 2.16. As for (ii), the analyticity of the "resonance" $E^{(+)}(\gamma)$ in the domain $\Delta_{\varepsilon}^{2}$ follows from Theorem 3.5 (see [11]). Thus the eigenvalue perturbation theory and standard remainder estimates [11] imply that $E^{(+)}(\gamma)$ is the Borel sum of order $\frac{1}{2}$ [3] of the associated expansion in any direction contained in $\Delta_{\varepsilon}^{1}$. To prove (ii) it is enough to note that $\Phi_{j}(g, \gamma)$ (where $g$ is fixed) has the same analyticity properties of $E^{(+)}(\gamma)$. Indeed, $h(g, z)(g>0, z \in \Gamma)$ is uniformly bounded on $\Gamma$ for small $g$, since by Theorem 3.6 it is convergent as $g \rightarrow 0^{+}$. We have $K(g, z) \rightarrow \exp (i(z+1) \pi)$, so that $h(g, z) \rightarrow \operatorname{cotan}((z+1) \pi / 2)$ uniformly on the compact set $\Gamma$ which does not contain the singular points $z=2 k+1, k=0,1, \ldots$ Moreover notice that $\psi(g)$ is bounded and $\psi(g) \stackrel{w}{\longrightarrow} \psi(0)$ as $g \rightarrow 0$. To prove (iii) it is enough to notice that

$$
b_{j k}(g)=(2 \pi i)^{-1} \oint_{\Gamma} h(g, z) z^{j}\left(A_{k}(g, z)+\overline{A_{k}(g, \bar{z})}\right) / 2 d z,
$$


where $A_{k}(g, z)=\left\langle\psi(g), R(0, z) \sum_{m=[k / 2]}^{k}\left(\begin{array}{c}k \\ k-m\end{array}\right)\left(2 x^{3} R(0, z)\right)^{2 m-k}\left(-x^{4} R(0, z)\right)^{k-m} \psi(0)\right\rangle$, and $a_{j k}(g)$ is given by $(4.21)$ with $h(g, z)$ replaced by 1 . Thus $b_{j k}(g)$ is directly computable as well as $a_{j k}(g)$ if we approximate in $(4.21) h(g, z)$ by $\tilde{h}(g, z)=h(0, z)=$ $\operatorname{cotan}((z+1) \pi / 2)$. In this approximation we only have to compute terms of the type $\left\langle\psi(g), \psi_{m}\right\rangle$, where $(\psi(g))(x)=\psi_{n}(x)+\psi_{n}\left(g^{-1}-x\right)$. Then

$$
\begin{aligned}
\left\langle\psi(g), \psi_{m}\right\rangle= & C \exp \left(-(2 g)^{-2}\right) \int_{-\infty}^{\infty} e^{-x^{2}} H_{n}\left((2 g)^{-1}-x\right) H_{m}\left((2 g)^{-1}+x\right) d x \\
& +\left\langle\psi_{n}, \psi_{m}\right\rangle=\exp \left(-(2 g)^{-2}\right) P_{n+m}\left(g^{-1}\right)+\delta_{n, m},
\end{aligned}
$$

where $0 \leqq m \leqq 3 k+n$. Notice that the approximation $h(g, z) \rightarrow h(0, z)$ does not destroy the summability properties discussed above and the sum is obviously given by the same expression (4.18) with $h(0, z)$ in place of $h(g, z)$. The same happens in the case of better approximations of $h(g, z)$ obtained by semiclassical methods.

Acknowledgement. It is pleasure to thank Professor Sandro Graffi for his interest in this work and useful discussions.

\section{References}

1. Brézin, E., Parisi, G., Zinn-Justin, J.: Perturbation theory at large orders for potentials with degenerate minima. Phys. Rev. D16, 408 (1977)

2. Caliceti, E., Graffi, S., Maioli, M.: Perturbation theory of odd anharmonic oscillators. Commun. Math. Phys. 75, 51 (1980)

3. Caliceti, E., Grecchi, V., Maioli, M.: The distributional Borel summability and the large coupling $\Phi^{4}$ lattice fields. Commun. Math. Phys. 104, 163 (1986)

4. Caliceti, E., Grecchi, V., Maioli, M.: Erratum to "The distributional Borel summability and the large coupling $\Phi^{4}$ lattice fields". Commun. Math. Phys. (in press)

5. Crutchfield II, W. Y.: No horn of signularities for double-well anharmonic oscillators. Phys. Lett. 77B, 109 (1978)

6. Graffi, S., Grecchi, V.: Resonance in stark effect and perturbation theory. Commun. Math. Phys. 62, 83 (1978)

7. Graffi, S., Grecchi, V.: The Borel sum of the double well perturbation series and the Zinn-Justin conjecture. Phys. Lett. 121B, 410 (1983)

8. Graffi, S., Grecchi, V., Harrell II, E. M., Silverstone, H. J.: The 1/R expansion for $H_{2}^{+}$: analyticity, summability and asymptotics. Ann. Phys. 165, 441 (1985)

9. Graffi, S., Grecchi, V., Simon, B.: Borel summability: Application to the anharmonic oscillator. Phys. Lett. 32B, 631 (1970)

10. Herbst, I. W., Simon, B.: Some remarkable examples in eigenvalue perturbation theory. Phys. Lett. 78B, 304 (1978)

11. Reed, M., Simon, B.: Methods of modern mathematical physics, Vol. IV. New York: Academic Press 1978

12. Sibuya, Y.: Global theory of a second order linear ordinary differential equation with a polynomial coefficient. Amsterdam: North-Holland 1975

13. Simon, B.: Coupling constant analyticity for the anharmonic oscillator. Ann. Phys. (NY) 58, 76 (1970)

14. Simon, B.: Large orders and summability of eigenvalue perturbation theory: A mathematical overview. Int. J. Quant. Chem. 21, 3 (1982)

15. Vock, E., Hunziker, W.: Stability of Schrödinger eigenvalue problems. Commun. Math. Phys. 83, 281 (1982) 